Running head: PERSONALITY AND OSTRACISM

Who gets ostracized?

A personality perspective on risk and protective factors of ostracism.

Selma C. Rudert ${ }^{1}$, Matthias D. Keller ${ }^{2}$, Andrew H. Hales ${ }^{3}$, Mirella Walker ${ }^{2}$, \& Rainer Greifeneder $^{2}$

${ }^{1}$ University of Koblenz and Landau, Germany

${ }^{2}$ University of Basel, Switzerland

${ }^{3}$ University of Virginia, USA

Author Note

This research was supported by a grant of the Research Fund for Junior Researcher of the University of Basel to Selma C. Rudert. We would like to thank Christiane Büttner, Sebastiaan Huizinga, and Sophia Oelmaier for their assistance with programming, data collection and analysis.

Correspondence concerning this article should be addressed to Selma C. Rudert, Social Environmental and Economic Psychology, University of Koblenz and Landau, Fortstr. 7, D-4055 Landau, Germany, E-Mail: rudert@,uni-landau.de

Study 1 preregistration: https://aspredicted.org/nu78n.pdf

Study 2 preregistration: https://aspredicted.org/9fr2w.pdf

Study 3a preregistration: https://aspredicted.org/nt9z7.pdf

Study $3 \mathrm{~b}$ preregistration: https://aspredicted.org/pg53j.pdf 
Study 4 preregistration: https://aspredicted.org/gi5zq.pdf

Study 5b preregistration: https://aspredicted.org/s4ht9.pdf

Verbatim materials and data for Studies 1- 4 and Study 5b are accessible at https://drive.switch.ch/index.php/s/7YpjTkOe1OGjpEB. Materials and data for Study 5a and Study 6 cannot be made available due to copyright and data protection laws.

Draft version: August 22th, 2019

(C) 2019, American Psychological Association. This paper is not the copy of record and may not exactly replicate the final, authoritative version of the article. Please do not copy or cite without authors permission. The final article will be available, upon publication, via its DOI:

$10.1037 / \mathrm{pspp} 0000271$

To be cited as: Rudert, S. C., Keller, M. D., Hales, A. H., Walker, M. \& Greifeneder, R. (in press). Who gets ostracized? A personality perspective on risk and protective factors of ostracism. Journal of Personality and Social Psychology. 


\begin{abstract}
Ostracism, excluding and ignoring others, results from a variety of factors. Here, we investigate the effect of personality on the likelihood of becoming a target of ostracism. Theorizing that individuals low in conscientiousness or agreeableness are at risk of getting ostracized, we tested our hypotheses within five pre-registered studies: Four experiments investigating participants' willingness to ostracize targets characterized by different personality traits and a reverse correlation face modelling study where we determined and subsequently validated the stereotypical face of an ostracized person. A survey study within a representative German data panel further corroborated our findings. In line with our hypotheses, persons low in conscientiousness or agreeableness provoke more ostracism intentions (Studies 1, 2, and 4), are more likely to be actually ostracized by others (Study 3), represent the stereotype of an “ostracizable” person (Study 5), and report experiencing more ostracism (Study 6). Effects remained stable even after controlling for likeability of the target (Study 2 and 4). Moreover, being described as negative on one personality dimension could not be compensated by being described as positive on the other (Study 4). In exploratory analyses, we further investigated the effects of openness to experience, neuroticism and extraversion. In sum, we find evidence that personality affects the likelihood of becoming a target of ostracism, and that especially low agreeableness and conscientiousness represent risk factors.
\end{abstract}

Keywords: ostracism, personality, agreeableness, conscientiousness, person perception 
Who gets ostracized?

Personality as a risk and protective factor of social ostracism.

Imagine working on a group project together with your team members, when you receive an email from a colleague who would be interested in joining your project. You could need some additional support and your colleague is generally a nice person. However, you know from past experience that this colleague often shows up late to meetings, is unreliable when it comes to deadlines, and the work output is often not as diligent as it is required. What would you answer your colleague, or would you even answer the email at all?

If you decide to decline your colleague's request to join the group project, or if you even ignore the email altogether, you have ostracized your colleague (Williams, 2009). Ostracism is a common occurrence in everyday life, such that on average, individuals report at least one or two incidences per day where they have ignored or excluded another person as well as have been ignored or excluded by another person (Nezlek, Wesselmann, Wheeler, \& Williams, 2012, 2015). Since ostracism is often a painful and threatening experience (Rudert \& Greifeneder, 2016; Rudert, Hales, Greifeneder, \& Williams, 2017; Williams, 2009), research investigating potential antecedents of ostracism is a highly important endeavor. Here we investigate perceived personality dispositions as a set of factors which can either put individuals at a higher risk of being ostracized, or protect them from becoming a target. Previous research on the relation between personality and ostracism has mainly investigated the self-reported experience of the target (Wu, Wei, \& Hui, 2011). Using self-reported experience generally precludes identifying the cause of the identified relationships. For instance, other individuals may decide to ostracize the target in response to behavioral manifestations of the target's personality that are picked up during interactions. Alternatively, the target's personality dispositions might affect the target's 
perceptions and interpretations of what exactly constitutes an instance of ostracism. Or there might even be reversed causal effects, such that the experience of ostracism affects the personality of the ostracized targets (Hales, Kassner, Williams, \& Graziano, 2016; Nielsen, Glasø, \& Einarsen, 2017). Here, we take a different approach to investigate the impact of personality dispositions by focusing on the motivations of those individuals who ostracize others (the so-called sources of ostracism). Using an experimental approach allows for causal interpretation of the effects of the targets' personality on the sources' intentions to ostracize.

\section{Motivated Ostracism}

To investigate potential antecedents of ostracism, we first consider factors that motivate groups and individuals to ostracize others. It is often assumed that the sources of ostracism are mean and act out of malicious motives and selfishness (Rudert, Sutter, Corrodi, \& Greifeneder, 2018), or that they simply do not like the ostracized target. However, in reality, this naïve assumption does not tell the full story. Individuals need to be careful if they choose to ostracize others, because in many situations, the general norm is to include others and to let them join in activities and groups if they wish to (Rudert \& Greifeneder, 2016; Wesselmann, Wirth, Pryor, Reeder, \& Williams, 2013). If sources ostracize others and violate this inclusion norm, they might easily end up being devalued by others or even punished for their behavior (Güroğlu, Will, \& Klapwijk, 2013; Over \& Uskul, 2016; Rudert, Ruf, \& Greifeneder, in press; Rudert et al., 2018; Will, Crone, van den Bos, \& Güroğlu, 2013). Consequently, many studies have demonstrated that individuals feel uncomfortable when they ostracize others without a plausible reason (Legate, DeHaan, Weinstein, \& Ryan, 2013).

As a consequence, in many situations individuals will only revert to ostracism when they have a strong motive to do so and/or can assume that others will approve of their decision to 
ostracize others. From an evolutionary perspective, Kurzban and Leary (2001) have argued that ostracism primarily occurs if individuals are perceived as bad exchange partners. This is the case, for instance, when (a) the ostracized target violates group norms, or (b) the ostracized target represents a burden for the sources (Kurzban \& Leary, 2001; Wesselmann, Wirth, Pryor, Reeder, \& Williams, 2015).

In cases of (a), the target has repeatedly violated either general social norms or specific group norms, for instance by acting particularly rude/uncooperative, or by ignoring specific agreements that the group established. Such norm violations threaten the harmony within a group as they create discord and increase the chance that other group members follow the negative example and start deviating from the norms as well, which would then destabilize the group and decrease cooperation (Ditrich \& Sassenberg, 2016; Kerr \& Levine, 2008; Scheepers, Branscombe, Spears, \& Doosje, 2002). Thus, individuals may choose to ostracize targets with the goal of punishing them and ultimately making them change their undesirable behavior, thereby protecting the group and its stability from being undermined by normlessness.

As for (b), some individuals may adhere to social norms, but nevertheless represent an inconvenient burden for a group. Groups often aim to achieve certain goals (McGrath, 1984), and some group members may be more useful in achieving these goals than others. And while it is a strength of groups that their members can complement each other and compensate for each other's weaknesses, a person that lacks either the skill or the motivation, or both, to make a meaningful contribution to a group effort can slow the group down substantially (Wesselmann, Williams, \& Wirth, 2014; Wesselmann et al., 2013). Groups and their members may thus be motivated to exclude a person that they perceive to be an underperformer and burdensome, in order to maintain the group's performance (Wesselmann et al., 2014). 
To summarize, an important reason for individuals to ostracize others is that ostracism serves as a social control mechanism, which ensures both the stability as well as the functionality of a group (Kurzban \& Leary, 2001). Whether an individual is likely to violate group norms, or turns out to be a burden for the group, might partly depend on characteristics of the group or the general situation. Some groups are more or less rigid in enforcing their norms (e.g., Gelfand et al., 2011) or have differences in proficiency levels and expectations for members. For instance, a player might easily be excluded from a professional football team for not meeting the high standards regarding performance and/or discipline, whereas at the same time, the same player might be well accepted in a group that plays only occasionally for recreational purposes. However, there might also be general characteristics of individuals which makes them more or less likely to be excluded from groups, and independent of the particular social context. In what follows, we discuss how an individual's personality can affect the likelihood that they will be ostracized.

\section{Dispositional Influences on the Likelihood of Becoming a Target of Ostracism}

In our research, we focus on the Big Five dimensions of personality, namely conscientiousness, agreeableness, neuroticism, openness to experience, and extraversion (Costa \& McCrae, 1992). Studies that have investigated relations between the Big Five and workplace harassment or workplace ostracism have found negative correlational relationships with conscientiousness, agreeableness, and extraversion, as well as a positive correlation with neuroticism (Nielsen et al., 2017; Wu et al., 2011). In addition to being confined to a workplace setting, however, most of the existing studies were based on self-reports of the targets and thus do not allow for causal conclusions. 
Nielsen and colleagues (2017) discuss three different ways to account for the correlation between target personality and the self-reported experience of workplace harassment, which are also likely to apply to ostracism experiences in general: First, via a target-behavior mechanism, meaning that targets may provoke harassment via their own personality, which manifests in the behavior they show towards others (especially for highly visible traits like extraversion; Vazire, 2010). In terms of ostracism, this would be comparable to the mechanism outlined above, namely the sources excluding the targets intentionally because the targets' behavior suggests certain personality characteristics that make the targets appear to be bad exchange partners. Second, via negative perceptions, meaning that individuals with certain personality disposition, such as neuroticism, might be more likely to interpret negative events as harassment. In terms of ostracism, it has also been shown that the perceptions and interpretations of an ostracism episode are highly important as they can change the experience and subsequent reactions to ostracism (Downey, Mougios, Ayduk, London, \& Shoda, 2004; Rudert \& Greifeneder, 2016; Wirth, Lynam, \& Williams, 2010; Zadro, Boland, \& Richardson, 2006). Third, via a reverse causality mechanism, meaning that the targets' dispositions might change as a result of being exposed to harassment for a prolonged time (Nielsen \& Knardahl, 2015; Podsiadly \& Gamian-Wilk, 2017). Again, in terms of ostracism, the existence of such vicious circles has also been shown, with ostracized individuals often acting more disagreeable, defensive, aggressive, or withdrawn (Downey, Freitas, Michaelis, \& Khouri, 1998; Downey et al., 2004; Hales, Kassner, et al., 2016; Ren, Wesselmann, \& Williams, 2016; Twenge, Baumeister, DeWall, Ciarocco, \& Bartels, 2007). The present contribution mostly focuses on the target-behavior mechanism, with the aim of establishing which perceived target personality dispositions make the sources intentionally want to ostracize a target. Given this focus, we will limit our review and theorizing to contributions 
relevant for the target-behavior mechanism. In what follows, we discuss predictions separately for each of the Big Five traits.

Conscientiousness describes the tendency for individuals to act in ways that are efficient, organized, planful, reliable, responsible, and thorough (McCrae \& John, 1992). Several studies as well as meta-analyses show that conscientiousness is the strongest predictor of productivity and performance both in the job as well as in academic settings (Barrick \& Mount, 1991; Hurtz \& Donovan, 2000; O’Connor \& Paunonen, 2007; Poropat, 2009; Rothmann \& Coetzer, 2003). It seems that "individuals who exhibit traits associated with a strong sense of purpose, obligation, and persistence generally perform better than those who do not" (Barrick \& Mount, 1991, p. 18). As groups often pursue certain goals, individuals who are perceived as useful in reaching these goals are likely to be considered valuable group members. At the same time, and consistent with the saying that "a chain is only as good as its weakest link," an underperforming group member can slow the group down and undermine team performance. As a consequence, it has been demonstrated that group members who are burdensome and decrease the group's performance are more likely to become targets of ostracism (Wesselmann et al., 2014; Wesselmann et al., 2013). Given the strong link between conscientiousness and performance, it thus appears likely that individuals who are low in conscientiousness are at a higher risk of becoming targets of ostracism.

Agreeableness, one of the two personality dimensions that strongly relates to social interaction, describes the tendency for individuals to treat others in ways that are appreciative, kind, generous, forgiving, sympathetic, and trusting (McCrae \& John, 1992). Agreeableness is linked to prosocial motivation (Graziano \& Eisenberg, 1997) and thus, agreeable people are less likely to be uncooperative or violate social norms (Berry, Ones, \& Sackett, 2007; Graziano, 
Habashi, Sheese, \& Tobin, 2007; Kagel \& McGee, 2014). In contrast, this means disagreeable people are more likely to violate group norms, and threaten harmony and group cohesion, which should make them less trustworthy and more likely to become targets of ostracism. This process has been demonstrated by Hales, Kassner, and colleagues (2016) in a series of studies, showing both that self-rated agreeableness is negatively related to self-rated ostracism, but also that individuals report higher intentions to ostracize a person described as disagreeable.

Neuroticism describes the tendency to act in ways that are anxious, self-pitying, tense, touchy, unstable, and worrying (McCrae \& John, 1992). On the one hand, it is easy to see how individuals high in neuroticism may become a burden for a group, not necessarily because of their performance, but because they may require more attention and thus be a strain for group interactions as well (Milam, Spitzmueller, \& Penney, 2009). Even if group performance is not affected, the experience of interacting with a neurotic group member may be sufficiently unpleasant to cause people to exclude and ignore this person. On the other hand, individuals who are anxious and unstable may also be perceived as particularly vulnerable and thus in the need of protection of a group. Ostracizing a vulnerable person might be evaluated as particularly cruel and unfair by other group members or outsiders (Rudert, Reutner, Greifeneder, \& Walker, 2017). As a consequence, groups that ostracize a vulnerable person might risk the anger, devaluation, or even punishment of others (Rudert et al., in press; Rudert et al., 2018).

Openness to Experience describes the tendency to act in ways that are artistic, curious, imaginative, insightful, and original, and to have a wide range of interests (McCrae \& John, 1992). A person particularly open to experience may be perceived as an attractive and interesting interaction partner and useful for group performance (especially concerning the intellect facet of openness), at least if creative solutions are what a group is aiming for. On the other hand, 
openness is also connected to pursuing unusual ideas or demonstrating unconventional behavior, which may be perceived as deviations from group norms. Openness to experience may thus also pose a threat to group harmony and stability. Moreover, individuals high in openness are often drawn towards new situations and (social) contexts. Interestingly, irrespective of the presently discussed target behavior mechanism, this might put individuals high in openness more often at risk of being ostracized. Given this higher base rate, individuals particularly open to experience might be statistically more likely to be rejected or ostracized compared to individuals who are likely to follow more familiar patterns.

Extraversion is closely related to social interactions and describes the tendency to act towards others in ways that are active, assertive, energetic, enthusiastic, outgoing, and talkative fashion (McCrae \& John, 1992). Since extraversion is often perceived as a higher interest in social activities and interactions as well as a higher quantity of social interactions (Ashton, Lee, \& Paunonen, 2002), and since extraverts tend to be more popular among peers (Jensen-Campbell et al., 2002), it seems initially plausible to expect a negative correlation between extraversion and the risk of becoming a target of ostracism. However, there are several reasons why one may not expect such a direct link: First, similar to individuals high in openness, extraverts might be more likely to encounter social situations and initiate social interactions with new partners (Snyder \& Gangestad, 1982), which means that the base rate of situations in which these individuals stand the risk of being ostracized may be higher than for individuals who stick to their close social circle. Second, even though an introverted individual might be less assertive and initiate social connections less often, that does not imply that others would deliberately decide to ostracize them or exclude them from activities they wish to join, especially as society becomes more aware of the idea of accommodating introverts (e.g., Cain, 2013). Still, highly 
introverted individuals might be at risk to become a target of involuntary or oblivious ostracism (Lindström \& Tobler, 2018; Williams, 1997), or in other words, they might simply be overlooked on accident by others.

Pulling all these considerations together, from a theoretical perspective as well as from the empirical evidence, there are strong reasons to expect a negative effect of both conscientiousness and agreeableness on the risk of becoming a target of ostracism. We thus hypothesized that individuals who are low in conscientiousness are more likely to become targets of ostracism because they may be perceived as a burden for group performance, and that individuals who are low in agreeableness are more likely to become targets of ostracism, because they represent a threat to group harmony and group norms. Because theoretical predictions for openness, neuroticism, and extraversion are less clear-cut, we decided to look at these remaining Big Five dimensions in an exploratory fashion, and pre-registered our studies accordingly on AsPredicted.org.

\section{Overview of the Studies}

In the present set of studies, we argue for effects of perceived target personality on the likelihood of being ostracized by others that are due to a target-behavior mechanism, that is, the sources ostracize the targets because of their personalities. Consequentially, there should be a direct causal link from sources' perception of the target's personality to the likelihood of ostracizing the target. Studies 1 - 4 test for this causal effect of perceived target conscientiousness and agreeableness on ostracism intentions, while controlling for liking (Studies 2 and 4). We hypothesized that low conscientiousness increases the likelihood of becoming a target of ostracism. Moreover, replicating the findings of Hales, Kassner and colleagues (2016), we further hypothesized that low agreeableness increases the likelihood of 
becoming a target of ostracism. In an exploratory fashion, in Studies 1 and 2, we also investigate potential causal effects of the other three personality dimensions (extraversion, openness, neuroticism) on ostracism intentions, without a priori hypotheses. Finally, Study 4 specifically tests for an interaction between conscientiousness and agreeableness.

Study 5 investigates the effect of personality on ostracism intentions with a different, more subtle method, namely via face modeling. Research has demonstrated that there is a strong social consensus regarding how the face of a person with a certain personality appears (Walker, Schönborn, Greifeneder, \& Vetter, 2018; Walker \& Vetter, 2016) and that individuals also intuitively base their (moral) judgments upon these facial cues (Funk, Walker, \& Todorov, 2017; Rudert, Reutner, et al., 2017). Against this background, we expect a social consensus of how a person that is likely to be ostracized stereotypically appears, and that this consensus will bear similarity to the consensus for facial appearance of a person with certain personality characteristics (e.g., a careless and disagreeable person). We investigate this hypothesis in Study 5 , using a reverse correlation paradigm.

Finally, if there is a substantial effect of target personality on ostracism, one could expect to find an association between self-reported personality and self-reported feelings of ostracism that can be identified in the real world outside of the laboratory. There are some studies regarding ostracism and harassment specifically in the workplace that hint at such a relation (Nielsen et al., 2017; Wu et al., 2011), however, it appears important to go beyond this preliminary evidence and show that the hypothesized relations can be demonstrated a) independent of a specific context such as the workplace and b) in a nation-wide representative sample. In Study 6, we thus investigate the relationship of the Big Five Personality Dimensions with subjectively experienced ostracism in a representative, longitudinal panel (the innovation 
sample of the German Socio-Economic Panel; SOEP-IS). Specifically, we test whether (prospectively measured) conscientiousness and agreeableness negatively predict self-reported ostracism.

\section{Access to Materials and Data}

The verbatim materials and data for Studies 1- 4 and Study $5 \mathrm{~b}$ are accessible at https://drive.switch.ch/index.php/s/7YpjTkOe1OGjpEB. Materials and data for Study 5a and Study 6 cannot be made publicly available due to copyright and data protection laws; see the respective study for more information.

\section{Study 1}

In Study 1, we manipulated the personality of a presented target and measured the potential sources' intention to ostracize the target. We predicted that individuals would report higher ostracism intentions for targets who were described as either low in conscientiousness or agreeableness. It should be noted that ostracism that is perceived as malicious and unfair can easily result in devaluation and punishment by others (Rudert et al., in press; Rudert et al., 2018), and also that people are aware of, and sensitive to, the pain of others who are ostracized (Coyne, Nelson, Robinson, \& Gundersen, 2011; Masten, Eisenberger, Pfeifer, \& Dapretto, 2010; Wesselmann, Bagg, \& Williams, 2009). Thus, ostracism will usually not be used light-heartedly. Specifically, individuals will likely ostracize others when they feel they have a valid reason to do so, namely if they have to protect themselves and their group from bad exchange partners (Kurzban \& Leary, 2001). Consequentially, we predicted that rather than following a strictly linear function, intentions to ostracize would increase specifically as a function of low conscientiousness as well as low agreeableness, compared to both high conscientiousness/agreeableness as well as a neutral control condition. 


\section{Method}

The study as well as all the subsequent experimental studies have been approved as part of the frameworks "Why do we ostracize others? Motives for social exclusion in groups" and "Faces" by the Institutional Review Boards of the Faculties of Psychology, University of Basel (011-17-1 and 034-15-3) and University of Koblenz-Landau (180_2019), and conform to recognized standards written in the Declaration of Helsinki.

Participants and design. Participants were recruited online from Prolific Academic (US Americans only) for a payment of $£ 0.40$. Based on the studies from Hales and colleagues (2016), we had initially calculated the sample size such as to detect a large-sized main effect of each personality dimension on participants' ostracism intentions $(f=.40$, power $=.80$, required $n=$ 304). However, data analysis from a pilot study showed that while we detected an effect of agreeableness with a comparable effect size as Hales and colleagues did, this effect was much larger than the effect of any other personality dimension. As a result, statistical power was likely too low to detect effects on the other dimensions that appeared to be more of a medium size. Moreover, some of the manipulation checks for our initial personality descriptions of the target person were not satisfactory. We thus slightly re-phrased some of the descriptions, which were then pre-tested separately, this time performing adequately. Additionally, based on these initial findings we re-calculated the sample size, this time to detect medium-sized main effects $(f=.25$, power $=.80$, required $n=579)$. Adding a buffer of 25 percent, we ran another, adequately powered study with a different sample of 801 participants on Prolific Academic, excluding all participants who indicated that their data should not be used (79) as well as participants who already participated in one of the pilot studies (7). The final sample thus consisted of 715 participants (335 females, 3 no specified gender; $M_{\mathrm{age}}=33.65, S D=11.83$ ). Here, we report only 
the data of the second, adequately powered sample. The study was a between-subject design, thus participants were randomly assigned to one out of eleven conditions (conscientiousness high vs. low; agreeableness high vs. low; neuroticism high vs. low; openness high vs. low; extraversion high vs. low; and a control condition). The study was preregistered on AsPredicted.org, see https://aspredicted.org/nu78n.pdf.

Materials and procedure. The study's procedure was adapted from Hales and colleagues (2016). Participants read a vignette that described a student named Mason. The basic version of each vignette contained no information about Mason's personality and was the same for all groups.

Mason is a 19 year old Sophomore student. He works as a part time job at a nearby restaurant. In his free time, he likes to watch movies, listen to music, and go outdoors. In a typical day, Mason goes to classes and afterwards spends some time on his computer. After dinner, he usually watches TV shows. His favorites are crime series, but he also enjoys quiz shows.

To make sure there would be no floor or ceiling effects of personality ratings of the vignettes basic version, we pretested the vignette with 15 participants on Prolific Academic (9 female; $\left.M_{a g e}=29.60, S D=7.50\right)$ who rated Mason on the Big Five personality dimensions (e.g., "Mason is disagreeable - agreeable”, 7-point scale). Mason was rated as fairly average on all dimensions (conscientiousness: $M=4.93, S D=1.39$; agreeableness: $M=4.60, S D=.99$; neuroticism $M=3.20, S D=1.42$; openness: $M=4.67, S D=.98$; extraversion: $M=3.53, S D=$ 1.25). In the actual study, control condition participants received the basic version of the vignette. In the remaining 10 experimental conditions, the vignettes were altered such that 
Mason was additionally described as either being high or low in one of the manipulated Big Five personality dimensions, see Table 1.

After reading the vignette, participants first answered a manipulation check about the respective personality dimension manipulated in their condition (e.g., "Mason is disagreeableagreeable", 7-point scale) as well as an attention question ("Mason likes crime series - hates crime series", 7-point scale). In the control condition, participants rated Mason on all five personality dimensions. Participants were then asked to imagine that Mason wanted to join a club they already belonged to and then reported their intention to ostracize Mason on a scale consisting of seven items (Cronbach's $\alpha=.89$; exemplary item: "I might find myself ignoring Mason", 1 = completely disagree, 5 = completely agree; Hales, Kassner, et al., 2016). After providing demographic information, participants were thanked and paid.

\section{Results}

Manipulation checks. We tested the manipulations' success using five one-way ANOVAs with the manipulated personality dimension (high vs. control vs. low) as independent variables, and the respective personality question as dependent variable. All ANOVAs revealed significant effects on the manipulated trait (conscientiousness: $F(2,197)=286.51, p<.001, \eta^{2}=$ .74 ; agreeableness: $F(2,198)=134.07, p<.001, \eta^{2}=.58$; neuroticism: $F(2,194)=142.00, p<$ $.001, \eta^{2}=.59$; openness: $F(2,197)=51.59, p<.001, \eta^{2}=.34$; extraversion: $F(2,186)=60.85, p$ $\left.<.001, \eta^{2}=.40\right)$. Bonferroni-corrected post-hoc analyses showed that for all five dimensions, the high condition was significantly greater than the control condition, and the low condition was significantly smaller than the control condition, largest $p=.003$. See Table 2 for descriptive statistics. 
Dependent variables. Five one-way $\mathrm{ANOVAs}^{1}$ (manipulated personality dimension: high vs. control vs. low) on intentions to ostracize Mason revealed significant differences for conscientiousness, $F(2,197)=38.18, p<.001, \eta^{2}=.28$; agreeableness, $F(2,198)=37.38, p<$ $.001, \eta^{2}=.27$; neuroticism, $F(2,194)=11.45, p<.001, \eta^{2}=.11$; and openness, $F(2,197)=5.31$, $p=.006, \eta^{2}=.05$. Manipulating extraversion did not predict intentions to ostracize, $F(2,186)=$ $0.01, p=.986, \eta^{2}<.01$

Bonferroni-corrected post-hoc tests for conscientiousness, agreeableness, neuroticism, and openness revealed that participants reported a higher intention to ostracize targets who were careless, disagreeable, emotionally unstable, and close-minded compared to both the control group and the respective positive characteristic, largest $p=.016$, see Table 3 and Figure 1 for the descriptive statistics. In contrast, there were no significant differences in ostracism intentions for targets that were described as conscientious, agreeable, emotionally stable compared to the control group, smallest $p=.659$.

\section{Discussion}

In line with hypotheses, we find that sources of ostracism report stronger intentions to ostracize a target who is low (vs. neutral or high) in conscientiousness or agreeableness. This effect was specific to the dimensions' low level; there was no significant difference between the neutral control condition and a high trait expression on the respective dimension.

In an exploratory fashion, we also investigated the other three Big Five dimensions. A description of the target as high in neuroticism or low in openness also elicited intentions to ostracize; however, the effects were much smaller than the effects of low conscientiousness and disagreeableness. It should be noted that we cannot rule out the possibility that manipulating one personality dimension also changed participant's perception of other dimensions. In fact, 
spillover effects are likely given that the Big Five are naturally correlated with each other (Soto \& John, 2017) and thus it is reasonable that participants might infer some personality dimensions from others. We further address this issue in Studies 4 and 5.

While participants were more inclined to ostracize targets who were careless, disagreeable, emotionally unstable, and close-minded, it appears that being explicitly conscientious, agreeable, emotionally stable, and open-minded did not offer any additional protection. This does not appear to be due to a failure to detect differences between control condition and high levels of these traits; the manipulation checks uniformly showed that people perceived the personality differences. Given that the reported intention to ostracize others was low on average, the finding could potentially be due to a floor effect. However, the resulting pattern also aligns with our theoretical assumptions that individuals will not decide to ostracize others lightly, and will thus primarily ostracize others when they feel they have a valid reason to do so, such as the target being a particularly bad exchange partner.

\section{Study 2}

As a next step, we investigated more closely what drives the effect of personality on ostracism intentions. One plausible mediator is liking. Previous research has demonstrated medium to strong positive relations between how much a person is liked and how that person is rated on conscientiousness, agreeableness, openness, extraversion, and (in the opposite direction) neuroticism (Leising, Erbs, \& Fritz, 2010). In addition, Hales and colleagues (2016) also showed that disliking predicts ostracism intentions and found it to be a strong mediator of the relation between ostracism and agreeableness.

While liking is thus a plausible mediator for the effect of personality on ostracism, there are also reasons to expect that the relationship between personality and ostracism does not 
depend exclusively on liking (Hales, Kassner, et al., 2016). Study 2 thus had both the aim to replicate the findings from Study 1, as well as to test whether the effect of personality dispositions on ostracism is fully mediated by liking, or instead whether there is unique variance not explained by liking of the ostracized target.

\section{Method}

Participants and design. Participants were recruited online from Prolific Academic (US Americans only) for a payment of $£ 0.40$. Based on the same (adapted) power calculations used in Study 1, we recruited 809 participants on Prolific Academic, excluding all participants who indicated that their data should not be used (9). The final sample thus consisted of 800 participants (345 females, 3 no specified gender; $M_{\text {age }}=32.35, S D=11.38$ ). Participants were randomly assigned to eleven conditions (conscientiousness: high vs. low; agreeableness: high vs. low; neuroticism: high vs. low; openness: high vs. low; extraversion: high vs. low; and a control condition). For pre-registration, see https://aspredicted.org/9fr2w.pdf.

Materials and procedure. The study's procedure was similar to Study 1. After answering the questions about intentions to ostracize (Cronbach's $\alpha=.89$ ), participants answered how much they like Mason on a five-item scale (Cronbach's $\alpha=.87$; exemplary item: "Mason is likeable", 1 = completely disagree, 5 = completely agree; Hales, Kassner, et al., 2016).

\section{Results}

Manipulation checks. We tested the manipulations' success using five one-way ANOVAs with the manipulated personality dimension (high vs. control vs. low) as independent variables, and the respective personality question as dependent variable. All ANOVAs revealed significant differences with regard to the manipulation: conscientiousness: $F(2,217)=736.08, p$ $<.001, \eta^{2}=.87$; agreeableness: $F(2,215)=146.43, p<.001, \eta^{2}=.58$; neuroticism: $F(2,214)=$ 
88.14, $p<.001, \eta^{2}=.45$; openness: $F(2,216)=108.45, p<.001, \eta^{2}=.50$; extraversion: $F(2$, $214)=138.33, p<.001, \eta^{2}=.56$. Bonferroni-corrected post-hoc analyses testing the differences between a high and a low value on the respective dimension compared to the control group were all significant, all $p \mathrm{~s}<.001$. See Table 4 for descriptive statistics.

Dependent variables. Ostracism Intentions. Five one-way ANOVAs (manipulated personality dimension: high vs. control vs. low) on intentions to ostracize Mason revealed significant differences for conscientiousness, $F(2,214)=50.09, p<.001, \eta^{2}=.32$; agreeableness, $F(2,215)=69.18, p<.001, \eta^{2}=.39 ;$ neuroticism, $F(2,214)=5.50, p=.005, \eta^{2}=.05 ;$ and openness, $F(2,216)=4.77, p=.009, \eta^{2}=.04$. Manipulating Extraversion did not affect intentions to ostracize, $F(2,214)=0.43, p=.650, \eta^{2}<.01$. Again, Bonferroni-corrected post-hoc tests revealed that participants reported a higher intention to ostracize targets who were careless, disagreeable, emotionally unstable, and close-minded compared to a group in which the respective target was positively described, as well as compared to the control group, largest $p=$ .037. In contrast, there were no significant differences in ostracism intentions for targets described as conscientious, agreeable, emotionally stable, and open-minded compared to the control group, smallest $p=.891$. Thus, being explicitly positive on a personality dimension did not offer any additional protection compared to the unmanipulated control setting. See Table 5 for the descriptive statistics.

Liking. Conscientiousness, agreeableness, neuroticism, and openness also significantly affected how much participants liked Mason: conscientiousness, $F(2,214)=60.77, p<.001, \eta^{2}=$ .36 ; agreeableness, $F(2,215)=61.25, p<.001, \eta^{2}=.36$; neuroticism, $F(2,214)=12.52, p<$ $.001, \eta^{2}=.11$; and openness, $F(2,216)=15.48, p<.001, \eta^{2}=.13$. Extraversion did not affect liking, $F(2,214)=1.69, p=.187, \eta^{2}=.02$. 
Examining the pattern of means, it can be seen that liking was distributed more linearly than ostracism intentions (see Table 5 for the descriptive statistics). Bonferroni-corrected posthoc tests for conscientiousness showed that participants liked Mason better in the control group compared to when he was described as careless, $p<.001$, and even better when he was explicitly conscientious compared to the control group, $p=.046$. For neuroticism and agreeableness, Mason was liked less when he was described as emotionally unstable or disagreeable, largest $p=$ .006 , but not significantly more when he was described as emotionally stable or agreeable, smallest $p=.079$. For openness, Mason was liked more when described as open compared to the control group, $p=.003$, but not less when he was described as close-minded compared to control group, $p=.085$.

ANCOVA and Mediation. When controlling for liking, intentions to ostracize Mason remained significant for conscientiousness, $F(2,213)=16.08, p<.001, \eta^{2}=.13$ and agreeableness, $F(2,214)=24.27, p<.001, \eta^{2}=.19$; but not for neuroticism, $F(2,213)=1.60, p=$ $.205, \eta^{2}=.02$, and openness, $F(2,215)=2.65, p=.073, \eta^{2}=.02$. We additionally ran mediation analyses with PROCESS (Hayes, 2013) using 5,000 bootstrap estimates. Liking mediated the effect of conscientiousness on ostracism intentions, $b_{\text {indirect }}=-.23,95 \% \mathrm{CI}=[-.36 ;-.12]$, though the direct effect remained significant as well, $b_{\text {direct }}=-.32, p<.001,95 \% \mathrm{CI}=[-.45 ;-.17]$. Similarly, liking mediated the effect of agreeableness on ostracism intentions, $b_{\text {indirect }}=-.23,95 \%$ $\mathrm{CI}=[-.38 ;-.11]$, but again, the direct effect remained significant, $b_{\text {direct }}=-.39, p<.001,95 \% \mathrm{CI}$ $=[-.53 ;-.25]$. When running separate mediation analyses with neuroticism and openness as the predictor, only the indirect effects were significant, neuroticism: $b_{\text {direct }}=.09, p=.125,95 \% \mathrm{CI}=$ $[-.02 ; .20], b_{\text {indirect }}=.09,95 \% \mathrm{CI}=[.03 ; .16]$; openness: $b_{\text {direct }}=-.10, p=.084,95 \% \mathrm{CI}=[-.21$; $.01], b_{\text {indirect }}=-.05,95 \% \mathrm{CI}=[-.11 ; .00]$. 


\section{Discussion}

Study 2 fully replicated the results of Study 1: When Mason was described as either low in conscientiousness or low in agreeableness, participants reported stronger intentions to ostracize him. As in Study 1, high neuroticism and low openness also increased ostracism intentions while extraversion did not. Again, all detected differences were due to a negative description of the target compared to both the positive as well as the neutral control condition, and again this is not attributable to failing to detect the positive personality conditions as differing from the control condition. Interestingly, this pattern was different for liking: Liking increased as a linear function of the respective personality dimensions, such that individuals were liked the more conscientious, open, (and by trend, the more agreeable) they were described.

This difference between ostracism intentions and liking was also reflected in the finding that even after including liking as a control variable, the effects of agreeableness and conscientiousness on ostracism intentions remained significant. Mediation analyses, as expected, showed liking to be an important mediator of the relation between personality factors and ostracism intentions. However, particularly for conscientiousness and agreeableness, significant variance could not be explained by how much participants liked Mason. For agreeableness, meaningful parts of this remaining variance are likely due to the tendency to distrust low agreeable targets as interaction partners - a factor identified to mediate the effect of agreeableness on intentions to ostracize, controlling for liking, in earlier research (Hales, Kassner, et al., 2016). Thus, it seems that while liking can explain some portion of the conscientiousness/agreeableness-ostracism link, it cannot entirely account for the relation on its own. This finding is reasonable when considering that there might be cases in which ostracism occurs independently of liking: For instance, a careless target might be well liked in principle, 
but still be excluded from a highly performance-oriented group. On the other hand, a person that is strongly disliked by others might not be ostracized because the group depends on that person or cannot afford to exclude one of its members.

\section{Study 3}

Studies 1 and 2 demonstrate that individuals report stronger ostracism intentions for individuals that are described as either low in agreeableness or conscientiousness. It remains unclear, though, whether these intentions would manifest in actual ostracism behavior. Thus, in Study 3 we used a group task paradigm in which individuals could choose to ostracize one of the group members to investigate whether individuals would actually exclude a disagreeable or careless person from the group. In the first leg (Study 3a) we examined the effects of conscientiousness, and in the second leg (Study 3b) we examined the effects of agreeableness.

In addition, we rendered the task more consequential by telling participants that all participating group members (including themselves) were eligible for a bonus payment that allegedly depended on overall group performance. Thus, participants' decision would have a) consequences for themselves, as they receive a higher bonus if their team performs well, and $b$ ) consequences for others, perhaps most evidently for the excluded person, as that person will not be part of the group and thus cannot receive a bonus. This parallels situations in real life where ostracized individuals often find themselves deprived of resources (Robinson, O’Reilly, \& Wang, 2013).

\section{Study 3a}

\section{Method}

Participants and design. Participants were recruited online from Prolific Academic (US Americans only) for a payment of $£ 0.60$ plus an additional bonus payment of $£ 0.30$. G*Power 
calculated a sample size of 317 participants to find a medium to small effect of target conscientiousness on the exclusion frequency $(w=.20, d f=2)$ with a power $=.90$. Oversampling slightly to account for possible data exclusions, we recruited 351 participants on Prolific Academic, excluding one participant who indicated that the data should not be used. The final sample thus consisted of 350 participants (178 females, 2 no specified gender; $M_{\text {age }}=33.61, S D$ $=11.69$ ). Participants were randomly assigned to three conditions (target conscientiousness: high vs. average vs. low). The study was preregistered on AsPredicted.org, see https://aspredicted.org/nt9z7.pdf.

Materials and procedure. All participants were informed that they would work on an online group task, and that they had been randomly chosen to be the leader of a group. As leader, they would have to choose the individuals that they want to work with. To make the choice more consequential for participants, they received the additional information that they, as well as all group members that they choose, would be eligible for a bonus payment up to a maximum amount of $30 \mathrm{p}$ per person (an amount sufficient to motivate performance, see Rudert et al., in press). The bonus would ostensibly depend on the overall performance of the group, not on the number of individuals who work on the task.

Participants were further presented with the names and descriptions of three potential group members. One piece of information was how each person ostensibly had scored on a conscientiousness questionnaire, specifically, whether they would fall into a "high," "average," or "low" cluster. Depending on the condition, the target person either scored high, average, or low on conscientiousness. The other two potential group members always fell in the "average" cluster. To provide some additional information, descriptions also included age and an “interesting fact" about the potential group members, such as "I have a twin brother." All filler 
information (interesting fact, age, name), as well as the order in which targets were introduced, were randomized.

Next participants were asked to make a categorical choice of whether they want to exclude any one of the three persons from the group. They could also choose to exclude none of the three persons. To facilitate analysis and account for expected low frequencies for the two control persons, choices were recoded into a binomial categorical variable (chose to exclude the target vs chose not to exclude the target). In addition, participants were asked to rate how conscientious they perceived each of the three persons on a 7 -point scale $(1=$ careless, $7=$ conscientious $)^{2}$. After providing demographic information, participants were thanked and paid. All participants received the bonus sum of $30 \mathrm{p}$ in addition to their regular compensation.

\section{Results}

A Pearson Chi-squared test showed a significant effect of target conscientiousness on exclusion frequency, $\chi^{2}(2)=138.64, p<.001, V=.45$. When the target was described as low in conscientiousness, $72 \%$ of the participants choose to exclude the target. In contrast, when the target was described as average (or high) in conscientiousness, only $14 \%$ (or $7 \%$ ) chose to exclude the target (Figure 2, Panel a). Similar to the results from Studies 1 and 2, especially low compared to average and high conscientiousness increased exclusion frequency (low vs. average: $\chi^{2}(1)=79.09, p<.001, V=.58$; low vs. high: $\left.\chi^{2}(1)=103.27, p<.001, V=.66\right)$. The difference between the average and the high condition was not significant, $\chi^{2}(1)=3.13, p=.077$, $V=.12$.

\section{Study 3b}

\section{Method}


Participants and design. As in Study 3a, we recruited 350 participants on Prolific Academic, excluding two participants who indicated that their data should not be used. The final sample thus consisted of 348 participants $\left(186\right.$ females; $\left.M_{\text {age }}=34.32, S D=11.93\right)$. Participants were randomly assigned to three conditions (target agreeableness: high vs. average vs. low). The study was preregistered on AsPredicted.org, see https://aspredicted.org/pg53j.pdf.

Materials and procedure. Materials and procedure were identical to Study 3a, except that information about the group members' personality related to agreeableness. For the manipulation check, participants were asked how agreeable they rated each of the three persons on a 7 -point scale $(1=$ disagreeable, 7 = agreeable $)$.

\section{Results}

Manipulation check. Ratings of the two control persons were averaged, Spearman's $\rho$ $=.79$. A $2 \times 3$ ANOVA (person: target vs. controls and condition: high vs. average vs. low) on perceived agreeableness showed a significant main effect of the person, $F(1,345)=25.38, p<$ $.001, \eta^{2}=.07$, the condition, $F(2,345)=117.63, p<.001, \eta^{2}=.41$, as well as a significant interaction, $F(2,345)=156.04, p<.001, \eta^{2}=.48$. Bonferroni-corrected simple main effects showed that in the average agreeableness condition, the target was perceived as less agreeable than in the high condition $\left(M_{\text {average }}=4.81, S D=1.14\right.$ vs. $\left.M_{h i g h}=6.02, S D=1.22\right)$, but as more agreeable than in the low condition $\left(M_{l o w}=2.54, S D=1.43\right)$, both $p<.001$. Rating of the control persons did not differ significantly across conditions $\left(M_{\text {high }}=4.98, S D=1.04, M_{\text {average }}=4.81, S D\right.$ $\left.=1.12, M_{l o w}=4.72, S D=1.05\right)$, smallest $p=.200$.

Dependent variable. Pearson's Chi-squared test showed a significant effect of target agreeableness on exclusion frequency, $\chi^{2}(2)=85.67, p<.001, V=.35$. When the target was described as low in agreeableness, $58 \%$ of the participants chose to exclude the target. In 
contrast, when the target was described as average (or high) in agreeableness, only $10 \%$ (or $12 \%$ ) chose to exclude the target (Figure 2, panel b). Similar to the results from Studies 1 and 2, especially low compared to average and high agreeableness increased exclusion frequency (low vs. average: $\chi^{2}(1)=58.06, p<.001, V=.50$; low vs. high: $\chi^{2}(1)=53.28, p<.001, V=.48$. The difference between average and high agreeableness was not significant, $\chi^{2}(1)=0.17, p=.677, V$ $=.03$.

\section{Discussion}

While Studies 1 and 2 investigated ostracism intentions, Studies $3 \mathrm{a}$ and $3 \mathrm{~b}$ show that individuals are also willing to put these intentions into actions. When a target person appears to be either low in conscientiousness or low in agreeableness, the target was excluded from the group by more than half of the participants. Replicating the general pattern of Studies 1 and 2, the detected differences were again due to the target scoring low compared to average or high on the respective personality dimension.

Importantly, participants were explicitly provided with the possibility to include everyone. Thus, a decision to leave out the target reflects more than just a rank-order preference within the group. Rather, it represents an actual decision to exclude. Moreover, participants were made aware that exclusion would have a financial disadvantage for the target, as an excluded target could not receive the group bonus. Thus, participants chose to exclude the target despite these negative consequences. Interestingly, while ostracism intentions in Studies 1 and 2 were low or moderate on average, in Study 3 the majority of participants chose to actually exclude a person that scored either low on agreeableness or low on conscientiousness. While this is possibly due to the differences in the paradigm, one can speculate whether in real life intentions are more susceptible to effects of social (un)desirability of ostracism. Individuals may be 
motivated to be inclusive and open in general, yet nevertheless decide to exclude undesirable others when actual stakes are involved. This effect might also be stronger if individuals are concerned that they might suffer a loss of resources (e.g. a lower group bonus) from including a disagreeable or careless person.

When comparing the effect sizes between Studies $3 a$ and $3 b$, the effect seems to be a little larger for conscientiousness than for agreeableness. Interestingly, in Study 2, we found the reverse, namely that the effect was largest for agreeableness. Possibly, this might be an effect of the respective context: As participants in Study 3 believed they had to solve a task together with others, they might have been in a stronger performance-oriented mindset, which might have led them to prioritize conscientiousness more strongly than agreeableness.

\section{Study 4}

Studies 1 - 3 investigated the effects of the Big Five on ostracism intentions independently from each other, which theoretically allows a focused test of each dimension. However, in real life, people we encounter differ from each other along more than just one personality trait, and thus the question arises how different traits may interact with one another. Here we examine the potential interaction between the traits of primary interest in this research, conscientiousness and agreeableness.

There are at least two possible ways in which conscientiousness and agreeableness could operate on ostracism intentions. First, one could expect that the more positive characteristics individuals have, the less likely they are, linearly, to be ostracized. In this case we would expect the traits not to interact but to combine in an additive way. That perspective would further imply that a personality disposition that is perceived as positive can (linearly) compensate for another 
personality dimension perceived as negative (Kervyn, Yzerbyt, Demoulin, \& Judd, 2008), such that a person is less likely to be ostracized if there is at least some good in her.

Yet it is also conceivable that the combined effect of the two personality dimensions follows more of a non-linear function. This is because individuals might go beyond the information that is given, evaluating a person who is being described as negative on one personality dimension as more negative in general (a negative halo effect, Nisbett \& Wilson, 1977). Moreover, given the importance of negative information in impression formation, scoring negatively on one personality dimension may not be fully compensated by scoring similarly positively on another. Consequentially, one could predict that positive information regarding one dimension may not fully compensate for negative information regarding another, resulting in an interaction effect between the two personality dimensions.

To test these competing predictions, in Study 4, we investigated the interaction of perceived conscientiousness and agreeableness on ostracism intentions. Since we reasoned that both conscientiousness and agreeableness are highly central for a person to be perceived as a good exchange partner (e.g., Fiske, Cuddy, \& Glick, 2007), we expected that being negative on one personality dimension cannot be compensated by being positive on the other. Thus, we predicted an interaction effect between conscientiousness and agreeableness such that the effect of either dimension on ostracism intentions is stronger if the target is described as positive (vs. negative) on the respective other dimension. In other words, a target must be at least minimally conscientious or minimally agreeable, in order to avoid ostracism and to allow for variation in the respective other trait to affect ostracism intentions.

As we do not wish to frame one personality dimension as more important than the other, we refrain from theoretically assigning one to the role of the moderator. However, as we realize 
that this makes our framing of the interaction rather non-specific, we would thus like to offer one example about how to think about it more specifically: We predict that the effect of conscientiousness on ostracism intentions is stronger if the target is described as at least minimally agreeable. In other words, if a partner cannot be trusted to cooperate, their level of competence should play less of a role in any decision to ostracize them, whereas if they are a trustworthy partner, their ability to contribute to a group should have more bearing on whether or not to include them. Methodologically speaking, we thus expected an interaction pattern in which the effect of conscientiousness is either not present ("knock out interaction") or is significantly reduced when a target is disagreeable.

\section{Method}

Participants and design. Participants were recruited online from Prolific Academic (US Americans only) for a payment of $£ 0.40$. The previous studies showed that we could expect large effect sizes for the main effect of both conscientiousness and agreeableness on ostracism intentions $(f=.40$, power $=.80$, required $N=52)$. As we intended to test for an interaction effect with a meaningful effect size, we based our calculations on recent power analysis recommendations for interactions with a so called "knockout pattern," (i.e. four times the sample size that has been used to show the original main effect; Giner-Sorolla, 2018; Simonsohn, 2014). Thus, we concluded that we need at least $N=208$; a sensitivity analysis with $\mathrm{G} *$ Power confirmed that this would allow us to detect an interaction effect of $f=.20$ with a power $=.80$. Oversampling slightly to account for possible data exclusions, we collected a sample of 232 participants on Prolific Academic, excluding all participants who indicated that their data should not be used ( 1 person). The final sample thus consisted of 231 participants (108 females; $M_{\text {age }}=34.43, S D=11.83$ ). Participants were randomly assigned to a 2 (conscientiousness: high 
vs. low) x 2 (agreeableness: high vs. low) between-subject design. The study was preregistered on AsPredicted.org, see https://aspredicted.org/gi5zq.pdf.

Materials and procedure. The study's procedure was similar to Studies 1 and 2. Participants read the vignette about Mason, who was either described as high vs. low in conscientiousness and high vs. low in agreeableness (no control condition). The order of personality descriptions was counterbalanced, such that some participants read about agreeableness first, and others read about conscientiousness first. Participants then answered the manipulation check about conscientiousness and agreeableness as well as the attention question about how much Mason liked crime series, reported their intention to ostracize Mason (Cronbach's $\alpha=.92$ ) as well as how much they liked Mason (Cronbach's $\alpha=.94)$. After providing demographic information, participants were thanked and paid.

\section{Results}

Manipulation checks. Participants in the high conscientiousness condition perceived Mason to be more conscientious than participants in the low conscientiousness condition, $F(1,227)=658.12, p<.001, \eta^{2}=.74\left(M_{\text {high }}=5.94, S D=1.59\right.$ vs. $\left.M_{\text {low }}=1.54, S D=1.11\right)$.

Moreover, participants in the high agreeableness condition perceived Mason to be more agreeable than participants in the low agreeableness condition, $F(1,227)=378.45, p<.001, \eta^{2}=$ $.63\left(M_{\text {high }}=5.94, S D=1.25\right.$ vs. $\left.M_{l o w}=2.56, S D=1.50\right)$. There was also a significant, though substantially smaller, effect of each personality dimension on the other, such that participants perceived Mason to be more agreeable when he was described as conscientious (vs. careless), $F(1,227)=22.52, p<.001, \eta^{2}=.09\left(M_{\text {high }}=4.10, S D=2.58\right.$ vs. $\left.M_{\text {low }}=2.56, S D=1.50\right)$, and more conscientious when he was described as agreeable (vs. disagreeable), $F(1,227)=22.42, p<$ 
$.001, \eta^{2}=.09\left(M_{h i g h}=4.67, S D=2.11\right.$ vs. $\left.M_{l o w}=3.85, S D=2.19\right)$. There were no significant interactions, smallest $p=.135$.

Dependent variables. A two-way ANOVA (conscientiousness: high vs. low and agreeableness: high vs. low) on intentions to ostracize Mason revealed significant main effects of conscientiousness, $F(1,227)=48.26, p<.001, \eta^{2}=.18$, and agreeableness, $F(1,227)=173.33, p$ $<.001, \eta^{2}=.43$, replicating the finding that both low conscientiousness and low agreeableness increase ostracism intentions. Moreover, the hypothesized interaction was significant, $F(1,227)=$ 12.64, $p<.001, \eta^{2}=.05$, see Figure 3. When Mason was described as agreeable, the effect of conscientiousness was larger, $F(1,227)=55.38, p<.001, \eta^{2}=.20\left(M_{C_{-} \text {Low }}=2.33, S D=.85\right.$ vs. $\left.M_{C_{-} H i g h}=1.34, S D=.38\right)$, than when Mason was described as disagreeable, $F(1,227)=5.73, p$ $=.018, \eta^{2}=.03\left(M_{C_{-} \text {Low }}=2.91, S D=.77\right.$ vs. $\left.M_{C_{-} \text {High }}=3.23, S D=.76\right)$. Alternatively, when Mason was described as conscientious, the effect of agreeableness was larger, $F(1,227)=$ $138.00, p<.001, \eta^{2}=.38$, than when Mason was described as low in conscientiousness, $F(1$, $227)=46.79, p<.001, \eta^{2}=.17$. In sum, intentions to ostracize Mason were highest when he was described negative on both personality dimensions, but being positive on either one of the two personality dimensions could not compensate for being negative on the other.

This pattern became more pronounced when controlling for liking. All main effects and interactions remained significant (conscientiousness: $F(1,226)=8.52, p=.004, \eta^{2}=.04$, agreeableness: $F(1,226)=5.24, p=.023, \eta^{2}=.02$, agreeableness x conscientiousness: $F(1,226)$ $=4.91, p<.001, \eta^{2}=.02$ ). When looking at the simple main effects, results slightly differ with respect to the significance level: When Mason was described as agreeable, low (vs. high) conscientiousness significantly increased ostracism intentions, $F(1,226)=12.21, p=.001, \eta^{2}=$ .05. In contrast, when Mason was described as disagreeable, low (vs. high) conscientiousness 
had no effect, $F<1$. Alternatively, when Mason was described as conscientious, low (vs. high) agreeableness significantly increased ostracism intentions, $F(1,226)=8.42, p=.004, \eta^{2}=.04$. However, when described as low in conscientiousness, low (vs. high) agreeableness had no effect, $F<1$.

There was an unexpected significant main effect of presentation order, $F(1,223)=5.24, p$ $=.009, \eta^{2}=.03$, namely that participants reported higher ostracism intentions when the information about agreeableness was presented first compared to second $(M=2.59, S D=1.03$ vs. $M=2.32, S D=.97)$. However, all interactions of order with the manipulated personality dimensions were not significant, smallest $p=.197$.

\section{Discussion}

Study 4 tested the interactive effect of conscientiousness and agreeableness on ostracism intentions. As predicted, the negative effect of being low on either dimension was stronger if the target was described as positive (vs. negative) on the other dimension. For instance, the negative effect of low conscientiousness was stronger when the target was described as agreeable (vs. disagreeable). When controlling for liking, ostracism intentions for disagreeable targets did not significantly differ based on whether they were described as conscientious or careless. But for agreeable targets, conscientiousness remained an important factor for intentions to ostracize, even after accounting for liking. The same pattern was true when framing the interaction the other way around.

This finding is in line with the strong significance of negative information in impression formation that has repeatedly been demonstrated in the literature on the negativity bias (Fiske, 1980; Skowronski \& Carlston, 1989). From the utilitarian perspective that ostracism ultimately serves the goal to eliminate bad exchange partners, it appears logical that being highly negative 
on one dimension cannot be sufficiently compensated by being positive on the other: Ultimately, an agreeable but careless individual might be just as problematic and troublesome for a group as a reliable but socially disruptive individual. The joint working of both the intent as well as the capability of a person is also highlighted in related models, such as the Stereotype Content Model (Fiske et al., 2007).

Another interpretation of our findings could be that the results are in fact due to some kind of a negative halo effect, as the manipulation checks show that a person that is described as disagreeable is also perceived as less conscientious and vice versa. Thus, the fact that there is not an additive effect of personality dimensions on ostracism intentions could be due to participants negatively adjusting their perception of Mason's entire personality when learning that he has one negative characteristic. Given that agreeableness and conscientiousness are correlated with each other (Soto \& John, 2017), it is not surprising that descriptions of one trait colored impressions of the other. However, because the main effects on the target traits were much larger than the spillover effects, it is parsimonious to conclude that the observed pattern is mainly due to the unique contribution of each independent trait.

Taken together, Studies 1 - 4 show that individuals report higher ostracism intentions towards targets with specific personality dispositions. Particularly, and as hypothesized, low conscientiousness and agreeableness strongly affect ostracism intentions. The effects were partly mediated by liking, however, the effect of both conscientiousness and agreeableness remained significant even after controlling for liking. Exploratory analyses of the results in Studies 1 and 2 showed that high neuroticism and low openness were also associated with stronger ostracism intentions, and that this relation could be partially explained by differences in liking (Study 2).

\section{Study 5}


In Studies $1-4$, personality was manipulated directly via description of the target person, and ostracism intentions were assessed rather explicitly, namely by asking participants how likely it is that they would ostracize a specific person. Since explicit preferences are sometimes prone to biases (e.g., Paulhus \& Vazire, 2007), we sought to further demonstrate the effect of personality on ostracism intentions with a more subtle measure. To this end, we built on evidence demonstrating a strong cross-cultural social consensus in personality judgments from faces (Walker, Jiang, Vetter, \& Sczesny, 2011; Walker \& Vetter, 2016). Generally, research shows that people tend to agree that certain faces appear to convey higher or lower levels of the five personality dimensions (Walker et al., 2018; Walker \& Vetter, 2016). For example, perceivers tend to agree that a particular person's face appears more or less conscientious. Although these judgments are not necessarily externally valid (Olivola \& Todorov, 2010), this consensus allows for a different test of our hypotheses: Given a consensus about how, for instance, a conscientious or agreeable person looks, and given our findings that conscientiousness and agreeableness are reliably associated with the likelihood of being ostracized, one might expect that the facial stereotype of someone that is likely to be ostracized might also be perceived as appearing low in agreeableness and conscientiousness. To test this, we proceed in two steps: Study 5a uses an image classification task (e.g., Mangini \& Biederman, 2004; Dotsch et al., 2008) and a statistical face modeling technique (Walker \& Vetter, 2016) to find out and visualize how people mentally represent (the face of) a person who is likely to be ostracized. In Study $5 \mathrm{~b}$ we then present this face to another sample of participants to find out whether such a face is perceived to be low in conscientiousness and/or low in agreeableness.

\section{Study 5a}

\section{Method}


The Basel Face Model is copyright-protected and thus the data and material of Study 5a cannot be made public. However, the model can be used freely for non-commercial scientific research and accessed by filling out a request form https://faces.dmi.unibas.ch/bfm/index.php?nav=1-1-0\&id=details. For all further information regarding access to the model please contact mirella.walker@unibas.ch.

Participants and design. We collected data from 40 participants (18 female, 1 no specified gender, $\left.M_{a g e}=24.13, S D=6.97\right)$. Participants were recruited on the university campus and compensated with CHF 3 (Swiss Francs; about the same in US\$ at the time) and a small chocolate present. Participants were randomly assigned to one of two different stimulus sets (see Materials and Procedure). The image classification task and the subsequent demographic questionnaires, however, were the same for all participants.

Materials. To create the stimuli for the image classification task we used the Basel Face Model (Paysan, Knothe, Amberg, Romdhani, \& Vetter, 2009; Walker \& Vetter, 2016), a multidimensional statistical face space derived from 200 3D scans of real faces. The dimensions of this space describe the shape (e.g., length, roundishness) and color information (e.g., darkness, contrast) with maximum variability between the 200 faces. Every face can be represented as a point in this multidimensional space, describing its position on all dimensions of the face space. Vectors pointing from one face to another describe the difference between the two faces (for details see Vetter \& Walker, 2011).

Combining the logic of the classical reverse correlation approach (Mangini \& Biederman, 2004) with the face space approach (Stolier, Hehman, Keller, Walker, \& Freeman, 2018; Walker \& Keller, in press), we created pairs of faces by applying random noise (i.e., random vectors in the face space) to a base face (i.e., the average face of the Basel Face Model). For each stimulus 
set, we created 98 vectors randomly manipulating shape information in faces, and 98 vectors randomly manipulating color information. Each random vector was once added to, and once subtracted from the base face, resulting in 196 face pairs per set (i.e., 98 face pairs varying only in shape and 98 pairs varying only in color information; see Figure 4 for two exemplar pairs).

Procedure. We told participants before the task that there were many reasons to exclude another person, and, that there were no right or wrong answers. Participants were then asked to indicate as spontaneously as possible which one of the two persons they would prefer to exclude from a group, by pressing one of two keys on the keyboard. In each trial, participants were presented with two faces, aligned horizontally on the screen. Each participant was presented with one of the two stimulus sets, that is, 196 trials in total. In the first 98 trials, the two faces presented only varied regarding shape information, and in the second 98 trials, the two faces presented only varied regarding texture information. Trial order within the shape as well as within the texture blocks was random. After 49 trials there was a short break and participants were free to continue whenever they were ready. After finishing the image classification task, participants answered a German version of the justice sensitivity scale (Schmitt, Baumert, Gollwitzer, \& Maes, 2010). The scale was assessed for exploratory reasons unrelated to the present research question and will not be discussed further here. After finishing the questionnaire, participants were thanked and compensated.

\section{Results}

We calculated a vector indicating the mental representation of an ostracizable person's face. This was done by averaging all vectors underlying the faces that were selected for exclusion by participants, resulting in a single ostracism vector (for more details, see Keller, Reutner, Greifeneder, \& Walker, 2019; Stolier et al., 2018; Walker \& Keller, in press). This 
combined ostracism vector was added to the base face to visualize the mental representation of a person that individuals would rather ostracize (henceforth referred to as the ostracism facial stereotype). Similarly, the ostracism vector was subtracted from the base face to visualize the mental representation of a person that individuals would rather not ostracize (henceforth referred to as the anti-ostracism facial stereotype; additional studies from our lab that are unrelated to the present research question found that this mental representation is also equivalent to the face of a person one would actively include). See Figure 5 for the visual results.

\section{Study $5 b$}

Study $5 \mathrm{~b}$ investigates whether the association between perceived conscientiousness and agreeableness and ostracism found in Studies 1-4 will emerge without directly manipulating said dimensions, but instead with a more subtle measure, namely impression formation from faces. To do so, we tested whether the stereotypical face of a person individuals would ostracize is perceived as low in conscientiousness and agreeableness. We presented both the ostracism and the anti-ostracism facial stereotype created in Study 5a to a new sample of participants and had them rate the two faces on the Big Five personality dimensions, as well as various other measures detailed below. We hypothesized that the ostracism facial stereotype would be rated as lower in conscientiousness and agreeableness than the anti-ostracism facial stereotype.

\section{Method}

Participants and design. We calculated the sample size to detect a medium-sized main effect of the presented stimulus faces on target personality ratings $(d=.50$, power $=.90$, required $n=44)$. We thus recruited 52 students (27 female, $M_{a g e}=24.17, S D=4.43$ ) in the university cafeteria, who participated in the study for CHF 3.50 (about the same in US\$ at the time). The 
study used a within-subject design and was preregistered on AsPredicted.org, see https://aspredicted.org/s4ht9.pdf.

Materials and procedure. Participants were presented, in random order, with the two faces generated in Study 5a: the stereotypical facial representation of an ostracized person (the ostracism facial stereotype) and the stereotypical facial representation of a person not to be ostracized (the anti-ostracism facial stereotype). For each of the two stimulus faces, they were asked to indicate their spontaneous impression of that person. More specifically, they were asked to rate the depicted persons on the Big Five personality dimensions, using two items for each dimension with highest factor loading from the BFI-K (e.g., reversed agreeableness: "The presented person can be cold and distanced"; Rammstedt \& John, 2005; see Walker \& Vetter, 2016). The stimulus faces were further rated on the Big Two (Agency and Communion, Runge, Frey, Gollwitzer, Helmreich, \& Spence, 1981; the two items with the highest factors loadings were used, see Walker \& Vetter, 2016), as well as attractiveness, sympathy, trustworthiness, and dominance. Moreover, we asked, using two items each, whether the person would comply with social norms, appeared familiar, and is healthy (psychologically and physically). All ratings were made on 7-point Likert scale $(1=$ completely disagree; 7 = completely agree $)$.

\section{Results}

A 2 (stimulus face: ostracism facial stereotype vs. anti-ostracism facial stereotype) x 5 (personality dimension: conscientiousness vs. agreeableness vs. neuroticism vs. openness vs. extraversion) within-subject ANOVA revealed significant main effects for the stimulus face, $F(1,51)=58.02, p<.001, \eta^{2}=.53$, and the personality dimension, $F(4,48)=24.11, p<.001, \eta^{2}$ $=.67$, that were both qualified by a significant stimulus face x personality dimension interaction, $F(4,48)=10.05, p<.001, \eta^{2}=.46$, such that the type of face affected some personality ratings 
more than others. Bonferroni-corrected simple main effects revealed that the ostracism facial stereotype was evaluated to be less conscientious, $p=.001, d=.51(M=3.78, S D=1.15$ vs. $M$ $=4.52, S D=1.22)$, less agreeable, $p<.001, d=1.10(M=2.77, S D=1.27$ vs. $M=4.68, S D=$ $1.34)$, and less open to experience, $p<.001, d=.74(M=2.81, S D=1.21$ vs. $M=4.26, S D=$ 1.46), than the anti-ostracism facial stereotype. Facial ostracism manipulations did not significantly affect ascriptions of extraversion, $p=.086, d=.24(M=3.48, S D=1.29$ vs. $M=$ 3.95, $S D=1.25)$ or neuroticism, $p=.452, d=.14(M=4.65, S D=1.21$ vs. $M=4.46, S D=$ $1.23)$.

As for the other measures, the stimulus faces were rated similar in agency, $t(51)=-1.78$, $p=.081, d=.25(M=4.75, S D=1.32$ vs. $M=4.28, S D=1.13)$, but the ostracism facial stereotype was evaluated to be lower in communion, $t(51)=7.60, p<.001, d=1.08(M=3.0$, $S D=.90$ vs. $M=4.64, S D=1.33$ ). The ostracism facial stereotype was also rated as less sympathetic, $t(51)=8.90, p<.001, d=1.24(M=3.02, S D=1.04$ vs. $M=5.02, S D=1.26)$, less attractive, $t(51)=6.25, p<.001, d=.88(M=2.60, S D=1.24$ vs. $M=4.08, S D=1.63)$, less trustworthy, $t(51)=7.36, p<.001, d=1.02(M=2.98, S D=1.20$ vs. $M=4.75, S D=1.22)$, but more dominant, $t(51)=-4.57, p<.001, d=.63(M=4.77, S D=1.45$ vs. $M=3.37, S D=$ 1.39) compared to the anti-ostracism facial stereotype. In addition, the ostracism facial stereotype was rated as less likely to comply with social norms, $t(51)=6.14, p<.001, d=.85$ $(M=3.62, S D=1.19$ vs. $M=5.02, S D=1.16)$, as well as to be less familiar, $t(51)=5.08, p<$ $.001, d=.71(M=2.65, S D=1.47$ vs. $M=3.94, S D=1.61)$, less similar to the participants, $t(51)=4.10, p<.001, d=.57(M=2.00, S D=1.10$ vs. $M=2.96, S D=1.39)$, and less likely to be physically and psychologically healthy, $t(51)=4.67, p<.001, d=.66(M=4.62, S D=1.38$ vs. $M=5.50, S D=.98)$ than the anti-ostracism facial stereotype. 


\section{Discussion}

Study 5 tested the link between personality and ostracism intentions with a new subtle paradigm. In a first part (Study 5a) using an intuitive, non-deliberative forced choice image classification task, we generated an ostracism facial stereotype and its anti-face from vectors in the Basel Face Model (Paysan et al., 2009; Walker \& Vetter, 2016). In the second part (Study $5 b)$, these two faces were localized on the Big Five personality dimensions and additional measures (e.g., the Big Two personality dimensions) by a different sample of participants. As expected, the ostracism facial stereotype was rated as less conscientious and less agreeable than its anti-face. In addition, the person with the ostracizable face was also rated as less open, but similar with regard to extraversion and neuroticism. These distinct effects suggest that individuals have a more differentiated stereotype of an ostracizable face than merely that of a face that is perceived as either positive or negative. This ostracism stereotype is also in line with research that has demonstrated that the stereotypical image of a bully, a target that individuals may wish to ostracize, is that of a person low in agreeableness and conscientiousness (Pallesen, Nielsen, Mageroy, Andreassen, \& Einarsen, 2017).

Study 5 indicates that individuals not only associate low conscientiousness and/or agreeableness with the likelihood of being ostracized, but that there is a socially shared notion of the appearance of a person likely to be ostracized, and this notion has strong traits of low conscientiousness and low agreeableness. Importantly, the fact that the new methodology allowed us to assess personality traits associated with ostracism without making any explicit allusion to ostracism, powerfully attests to the robustness of our findings.

\section{Study 6}


Studies 1-5 present experimental evidence that individuals with certain personality characteristics are more likely ostracized, and also demonstrate that there is a common facial stereotype of an ostracized person that is linked to certain personality characteristics. However, a crucial question is whether such intentions are translated into behavior in real life, and thus whether the obtained results are ecologically valid. Assuming that the sources' intentions translate into behavior not only in research settings (as found in Study 3), but also in daily life, and taking into account that individuals are highly sensitive to experiences of ostracism (Rudert, Hales, et al., 2017; Williams, 2009), one should expect that individuals who are less conscientious or agreeable report experiencing ostracism more often. It should be noted that we are not first to address this important question. Previous studies on the ostracism/personality link, which focused on the workplace and used small and specific samples (Nielsen et al., 2017; Wu et al., 2011), have found relations between self-reported ostracism and conscientiousness, agreeableness, neuroticism, as well as openness. We aim to complement these findings by testing the hypothesized relations in a general context, with a large-scale, representative sample from the German Socio-Economic Panel (SOEP; Goebel et al., 2018). Despite its importance, such general evidence is lacking in the literature so far.

\section{Method}

The SOEP data is subject to data protection laws of the Federal Republic of Germany, thus, the data cannot be made public. However, free access is granted to all scientific researchers who sign a contract with the German Institute for Economic Research (DIW Berlin). Please see https://www.diw.de/en/diw_02.c.222829.en/access.html for access procedures or contact soepmail@diw.de. 
Sample. We used data from the 2015 wave of the SOEP, a representative longitudinal survey of German households with almost 20,000 participants who are surveyed on an annual basis. Specifically, the scales that are relevant for our research question were part of the survey given to the innovation sample (SOEP-IS), a subsample of the SOEP that specifically allows for testing new research questions. In sum, 2,745 individuals $\left(53 \%\right.$ female, $M_{\text {age }}=52.44, S D=$ 18.26, Range Year of Birth $=1919$ - 1998) answered the newly developed Ostracism Short Scale (OSS). Participants were nested within 1,718 households. Of these, 881 households (51.3\%) provided one participant, 695 households $(40.5 \%)$ provided two participants, and 142 households $(8.2 \%)$ provided three or more participants.

\section{Measures.}

The Ostracism Short Scale (OSS). To be able to contribute items to the nationwide representative SOEP-IS survey, we needed to provide a measurement for the frequency of ostracism that consists of very few items. To this end, we developed the Ostracism Short Scale (OSS) that was based on the Ferris scale (Ferris, Brown, Berry, \& Lian, 2008). The OSS is a four-item scale measuring the general subjective frequency that a person had felt ostracized within the previous two months. In particular, participants were asked (in German): "How often did you experience the following occurrences during the last two months?" with respect to: "Others ignored me", "Others shut me out from the conversation", "Others treated me as if I wasn't there", "Others did not invite me to activities". All items are rated on a 7-point Likert scale $(1=$ never, $7=$ always $)$. We pretested the OSS in a sample with 175 participants (123 female, 3 no specified gender, $M_{a g e}=26.33, S D=7.60$, Range $=18-79$, recruitment via the online pool of the German-speaking social psychology project "Forschung Erleben"). The OSS showed a high reliability (Cronbach's $\alpha=.87$; compared to e.g., $\alpha=.89$ of the original 10-item 
scale; see Ferris et al., 2008). Moreover, we tested for criterion validity by correlating the OSS with several related measures such as the Relatedness subscale from the Balanced Measure of Psychological Need Scale (Sheldon \& Hilpert, 2012), and loneliness (Hawkley, Duvoisin, Ackva, Murdoch, \& Luhmann, 2015; Luhmann \& Hawkley, 2016). Concurrent validity was achieved by correlating the OSS with the Satisfaction with Life Scale (Glaesmer, Grande, Braehler, \& Roth, 2011), the World Health Organization-5 Well-being index (Brähler, Mühlan, Albani, \& Schmidt, 2007), Need threat and mood (Rudert \& Greifeneder, 2016), as well as life satisfaction measures (Lang, Weiss, Gerstorf, \& Wagner, 2013; Schimmack, Krause, Wagner, \& Schupp, 2010). The OSS showed medium correlations (highest $r=.58$ with need threat and loneliness, lowest $r=-.34$ for life satisfaction in the next five years), see Table 6, which speaks to the scale's validity.

The Big Five. Within the SOEP, the Big Five were assessed with the short scale BFI-S (Gerlitz \& Schupp, 2005), which measures each of the five personality dimensions with three items using a seven-point Likert scale ( 1 = completely disagree, 7 = completely agree $)$.

\section{Results}

The zero-order correlations revealed that ostracism was negatively correlated with conscientiousness, $r=-.20, p<.001$, agreeableness, $r=-.18, p<.001$, extraversion, $r=-.19, p<$ .001 , and positively with neuroticism, $r=.20, p<.001$. Openness was not significantly related to ostracism, $r=.02, p=.445$. When we regressed ostracism on all five predictors simultaneously, we found negative relations with conscientiousness, $\beta=-.13, p<.001$, agreeableness, $\beta=-.13, p$ $<.001$, extraversion, $\beta=-.17, p<.001$, and a positive relation with neuroticism, $\beta=.16, p<$ .001. Interestingly, there emerged a positive relation between ostracism and openness, $\beta=.08, p$ $<.001$. The variance of ostracism explained by the Big Five was $R^{2}=.11$. 
To test for temporal stability, we performed a regression predicting ostracism reported in 2015 from the Big Five reported in 2013. Again, we found negative relations with conscientiousness, $\beta=-.12, p<.001$, agreeableness, $\beta=-.07, p<.001$, extraversion, $\beta=-.12, p$ $<.001$, and a positive relation with neuroticism, $\beta=-.06, p<.001$. There was no relation between ostracism and openness, $\beta=.01, p=.528$. The variance explained by the regression model was $R^{2}=.06$.

\section{Discussion}

The data from the SOEP-IS showed evidence for a general link between the Big Five personality dimensions and experienced ostracism. Individuals who perceive themselves as more conscientious, agreeable, emotionally stable, or extraverted reported being ostracized less often. These relations could be found when we predicted ostracism experience based on data assessed at the same point in time as well as based on data collected two years earlier, ruling out the specific measurement point at which participants took the survey as a possible explanation, and attesting to temporal stability. Given the representativeness of the sample for the underlying population, the data thus speak to the generalizability of the obtained results, at least within Germany, a relatively culturally independent nation (Markus \& Kitayama, 1991).

The present results partly dovetail with findings obtained by $\mathrm{Wu}$ and colleagues (2011). In particular, $\mathrm{Wu}$ and colleagues reported a survey with 208 employees in the petroleum industry in China, a relatively culturally interdependent nation (Markus \& Kitayama, 1991). They found negative relations of workplace ostracism with agreeableness and extraversion and a positive relation with neuroticism. Our observed relationships for the experience of ostracism from the SOEP conceptually align with the meta-analysis of Nielsen and colleagues (2017), who investigated the effect of target personality on the experience of workplace harassment. Similar 
to our findings, the meta-analysis showed positive relations of experienced workplace harassment with conscientiousness, agreeableness, and extraversion, as well as a negative relation with neuroticism, but none with openness.

The SOEP data represent an important final link in our argument, as it indicates that the effects that we demonstrated within the lab transfer, at least partly, to the real world. One should note that the strength of the associations differs from what was observed in the experimental studies. Particularly, while conscientiousness and agreeableness again robustly predicted ostracism, other predictors proved to be equally strong. In contrast to the experimental studies, in the SOEP, extraversion was a significant predictor and openness was not. One possible explanation is that due to the cross-sectional nature of the data, the obtained associations might be affected by several other processes (for example, highly open individuals may seek out more situations, including some where they encounter more ostracism). Moreover, there might be reversed causality processes, that is, ostracism affecting a target's personality. We discuss this in more detail within the General Discussion.

\section{General Discussion}

Ostracism can be caused by a variety of factors, one of which appears to be the personality characteristics of the ostracized target. The present manuscript examines the notion that targets may provoke ostracism as a result of their personality characteristics. We predicted and pre-registered our hypotheses that targets especially low in agreeableness and low in conscientiousness elicit ostracism. We found consistent evidence within six studies and across three different paradigms. Studies 1, 2, and 4 show that individuals report more ostracism intentions for targets that are described as disagreeable or careless, and Study 3 shows that these intentions also manifest in actual ostracism behavior. The effect was partially mediated by liking 
(Studies 2 and 4), but not additive, given that being described as disagreeable could not be fully compensated by being described as conscientious and vice versa (Study 4). We further found that there is a socially shared facial stereotype of how a person likely to be ostracized appears, which is that of a careless and disagreeable person (Study 5). Finally, when analyzing data from a representative survey (the SOEP-IS), self-reported agreeableness and conscientiousness reliably predicted experienced ostracism, even when ostracism was measured two years later (Study 6).

The obtained results constitute an important step in understanding the reasons why ostracism occurs in the first place - a question that has played a minor role in ostracism research so far. We believe this question to be a highly important one, given that to prevent ostracism, knowledge about its antecedents may prove highly useful. Of course, there are a variety of reasons why ostracism may occur, many of them are due to situational circumstances or are even purely incidental results of random selection mechanisms (Lindström \& Tobler, 2018). Moreover, different causes may operate at different levels of explanation; a target's personality is a relatively ultimate, or upstream cause of later ostracism, while a source's disliking - elicited by the target's personality- represents a more proximate, or downstream cause. As personality can be understood as accumulated behavior of a person over time (e.g., Fleeson, 2001), it is likely that it is at least one crucial reason for why some individuals are more prone to become targets of ostracism compared to others. While the experimental studies (Studies 1-5) shed light on one of the potential mechanisms by which personality might increase ostracism intentions and behavior of the sources, Study 6 demonstrates that the respective relations also exist in real life and can be shown in a representative sample. The studies were conducted with participants from three different countries, the United States (Studies 1 - 4), Switzerland, (Study 5), and Germany (Study 6), suggesting that the results generalize, at least within cultures that can be characterized 
as independent and individualistic (Hofstede, 1980; Markus \& Kitayama, 1991). However, as our findings dovetail with research conducted in interdependent or collectivist cultures such as China (Wu et al., 2011), this speaks to the generalizability and stability of our results across cultures and standard demographic variables.

\section{The Source Perspective: Motivations for Ostracism}

Within the scope of this manuscript, we mainly focused on the sources' perspective (Studies 1-5) and proposed two reasons why individuals would ostracize others: First, the target has violated social norms, which we reasoned to be the case for individuals perceived as disagreeable, and second, the target represents a burden to the group, which we reasoned to be the case for individuals perceived as low in conscientiousness. It should be emphasized that these reasons are not necessarily mutually exclusive, in fact, it is plausible that in some situations, they might even influence one another. For instance, a target that consistently violates group norms is likely to be perceived burdensome at some point. Inversely, a continuous underperformance might be interpreted as social loafing and a violation of a group's performance norms. This finding is also in line with the "negative halo effect" demonstrated in Study 4, showing that an individual described as low in conscientiousness was also perceived as less agreeable and vice versa. Rather than representing a methodological caveat, we believe that it is highly probable that individuals' assumptions of how certain personality dimensions are related are in line with actual correlations between the respective personality dimensions (Soto \& John, 2017).

Of course, aside from target personality there are other factors that might influence intentions and decisions to ostracize. One is the sources' personality. For instance, it has been shown that source agreeableness is negatively related to bullying behavior, thus, is appears plausible that it might also be related to ostracism (van Geel, Goemans, Toprak, \& Vedder, 
2017). But it is also possible that there might be interactions between source and target personality on intentions to ostracize. These interactions might be driven by biased perceptions of similarities and the self-image bias, namely that personality characteristics that a person possesses are weighed as more important and central (Lewicki, 1983). That is, a highly conscientious person who feels that conscientiousness is a very important trait might be more inclined to ostracize a target for being careless, while a person scoring low on conscientiousness may not pay much attention to conscientiousness information about others. Moreover, sources might refrain from ostracizing a potential target for a reason that would apply to themselves as well.

Another - and possibly even more relevant - moderator should be the situational context. In the present studies, we deliberately aimed to reduce context effects to a minimum and thus gave very little information about the group or its goals. In these rather context-free settings, we documented roughly comparable effects of agreeableness and conscientiousness. However, we strongly assume that when adding specific contextual information, the effect of the two dimensions on ostracism decisions is likely to shift. To illustrate, in a purely social context, such as a group of friends meeting in a bar, conscientiousness might be less important than agreeableness when deciding who to include or exclude. On the other hand, in a performance context, a careless person who reduces the group's performance might be readily excluded while a disagreeable person might not, if this person is especially competent and central for the success of the group. Likewise, there might be temporal effects, such that certain target personality traits might provoke ostracism immediately, while others might cause ostracism only after some time had passed (e.g., Paulhus, 1998). For instance, disagreeableness might show rather quickly leading individuals to promptly ostracize the troublemaker. In contrast, low conscientiousness 
might not be relevant until competition occurs, or may be excused as accidental slips at first. The latter might especially be the case when a new member joins an existing group and is not familiar with its procedures yet.

Consistent with this reasoning, differences in the documented effect sizes of the conducted studies hint at the importance of context: While in the studies using the Mason vignette (Studies 1, 2 and 4), agreeableness usually had a stronger effect than conscientious ness, and in the studies in which participants made a choice for an ostensible group task (Studies 3a and $3 \mathrm{~b}$ ), the conscientiousness information had a stronger effect than the agreeableness information. One possible reason for this is that participants paid more attention to conscientiousness information when they expected a performance task. However, as the studies did not directly compare context, at present this remains speculation only. Further research on contextual moderators as well as interactions with personality of the sources might clarify its effect on the relation between target personality and ostracism.

\section{Effects of Extreme Personality Dispositions}

Studies 1, 2 and 3 show that ostracism intentions and behavior mainly increase due to individuals having negative personality dispositions, such as being disagreeable or careless. Being positive on one personality dimension did not decrease ostracism further compared to the control group. One can speculate, though, that a person with an extreme personality disposition in a positive direction could also face an increased risk of ostracism. For instance, under certain situational circumstances, a highly conscientious top-performer can get bothersome and pose a threat to a group, as that person may make the rest of the group look less competent or threaten the position or status of single group members (Maner \& Mead, 2010). Moreover, obsessivecompulsive personality disorder (OCPD) has been described as an extreme, maladaptive form of 
conscientiousness (Samuel \& Widiger, 2011) that is characterized by perfectionism and an unhealthy preoccupation with order and organization. It is easy to see how such a person might become a burden for a group as well, and thus be at an increased risk of becoming a target of ostracism. This notion is also in line with research showing that individuals dislike others the more they perceive these others to have extreme dispositions (Koch, Imhoff, Dotsch, Unkelbach, \& Alves, 2016). Given that dislike partly mediated the link between personality and ostracism intentions, it is plausible that being extreme on a specific personality dimension might increase the risk of becoming a target of ostracism, too. However, such a potential negative effect of an extreme personality would probably only hold for some dimensions but not for others; in particular, it is less easy to imagine that a person could be ostracized for being too agreeable, given the importance of warmth in person perception (Koch et al., 2016).

\section{Neuroticism, Openness, and Extraversion}

This contribution focused on the effects of conscientiousness and agreeableness, as it was our impression that the existing literature allows for rather clear-cut predictions for these two traits. This impression was supported by the data collected. Despite this (pre-registered) focus, in Studies 1, 2, 5 and 6, we also assessed the remaining Big Five Personality Dimensions in an exploratory fashion. Although speculative and less clear, we would like to briefly discuss the different patterns we found for neuroticism, openness, and extraversion, and offer some potential explanations that might become the basis for confirmatory research on these traits.

Perceived neuroticism increased ostracism intentions in Studies 1 and 2, moreover, selfreported neuroticism in the SOEP-IS was positively related to self-reported ostracism.

Interestingly, in Study 5, neuroticism did not seem to be part of the facial stereotype of a person who is likely to be ostracized. One possible explanation for this is that in real life, individuals 
generally cannot reliably detect neuroticism (Vazire, 2010) and thus, neuroticism may not be a part of the facial ostracism stereotype. Alternatively, while highly anxious and nervous individuals might both be more sensitive to social ostracism as well as perceived as bothersome and thus be ostracized more often, there might be less of a social consensus to do so. As previous research has demonstrated, individuals are aware that ostracism is painful for the ostracized person (Legate et al., 2013; Masten et al., 2010; Wesselmann et al., 2009) and strongly disapprove of groups ostracizing individuals that can possibly not take care of themselves and depend on the protection of the group (Rudert, Reutner, et al., 2017). Thus, while ostracizing an emotionally unstable person may come with benefits for the group, it also carries the risk of social (disapproval) costs. Further research may fruitfully investigate whether the current facial findings generalize to explicitly expressed stereotypes as well.

Low openness increased ostracism intentions in Studies 1 and 2 and was also part of the facial ostracism stereotype in Study 5. However, in the representative data from the SOEP-IS (Study 6), self-reported openness was not significantly, or even positively, related to selfreported ostracism. There are at least two potential explanations for this finding: First, when asked directly, individuals might over-estimate the positivity of high openness. While typically perceived as a positive characteristic, openness is also linked to unconventionality and a tendency to disregard social norms (McCrae \& Sutin, 2009), which could be a reason for others to ostracize a very open individual. Second, while others might be more inclined to ostracize close-minded persons, close-minded persons may generally not approach new situations very often. As a consequence, close-minded individuals might be less likely to encounter situations in which they might experience ostracism. Individuals high in openness, instead, might seek unfamiliar environments that come with the risk of rejection more often (for example, parties 
populated mostly with peripheral social connections). These ecological effects may be reflected in the survey but not in our laboratory studies, thus offering one explanation for the difference between the negative effects we found in the experiments and the null, or even positive effect in the representative sample.

Finally, low extraversion did not predict ostracism in any of the experiments, nor was extraversion a part of the facial ostracism stereotype in Study 5. However, we found a substantial and stable negative relation between self-reported extraversion and self-reported ostracism in Study 6. A plausible explanation for this finding would be oblivious ostracism: introverted people might be overlooked more often and consequentially experience ostracism more often, even though the involuntary sources of ostracism might not have intentionally planned to ostracize them (Lindström \& Tobler, 2018; Williams, 1997).

\section{Methodological Considerations}

It should be noted that while all studies within the present contribution investigate the relation between ostracism and personality, they differ in their focus and the investigated perspective: Studies 1- 4 investigate the motivation and intentions of the sources to ostracize others as well as actual ostracism behavior, with personality of the target being manipulated and presented to participants. In daily life, people can detect certain traits more accurately than others. For example, extraversion, characterized by sociability and visible behaviors, tends to be accurately detected, while neuroticism, characterized by distressed internal mental states tends to be less accurately detected (Vazire, 2010). It follows then, that in real life, ostracism intentions might further be affected by how well individuals can detect certain traits, such as there could be stronger biases for traits which individuals can detect less well. Alternatively, it is also possible that individuals might be aware of their ability to detect certain traits and will be more careful to 
ostracize persons due to personality dimensions they can detect less well, as we have previously discussed for neuroticism.

Study 5 focuses on the stereotypical perception of individuals who become a target of ostracism. Although closely related to Studies 1-4, this study uses a subtle measurement and thus demonstrates that the suggested association between personality and ostracism is present even if individuals cannot easily discern what is measured.

Study 6 focuses on the perceptions and self-evaluation of the targets of ostracism. While agreeableness and conscientiousness were again related to ostracism, it appears likely that in this study, additional mechanisms aside from the motivation of the sources influence this relation. This assumption is also in line with differences in the detected patterns: In the experimental studies, conscientiousness and agreeableness were the strongest predictors of ostracism intentions, while in the SOEP-IS data, extraversion and neuroticism predicted ostracism more strongly. It is thus likely that the effects of extraversion and neuroticism are mainly due to other mechanisms than the target provoking ostracism.

Two of these alternative processes could be influences of target perception, that is, individuals with certain personality dispositions interpreting negative events as ostracism, and reversed causality, that is, individual's personality changing as an effect of repeatedly being ostracized (Nielsen et al., 2017). In real life, it is highly likely that the three mechanisms (target provocation, target perception, and reversed causality) are strongly interconnected in vicious circles: For instance, individuals who tend to interpret even minor incidents as intentional ostracism by others may behave in a manner that provokes actual ostracism, which would then confirm their beliefs. Similar processes have been shown for rejection sensitivity (Downey et al., 
1998; Downey et al., 2004), a disposition that is closely related to neuroticism, and for agreeableness (Hales, Kassner, et al., 2016).

\section{Practical Implications}

As research on ostracism has mainly focused on the target's perspective, most attention has been given to the question of how to improve and assist target recovery following ostracism (e.g., Hales, Wesselmann, \& Williams, 2016; Rudert, Hales, et al., 2017; see Timeo, Riva, \& Paladino, 2019 for an overview). Here, we focus on a different perspective, namely reasons that motivate the sources to ostracize a person, such as the target being disagreeable or careless and thus being perceived as a bad exchange partner. We believe that a deeper understanding about these processes is essential if one's goal is to prevent ostracism from occurring in the first place instead of merely dealing with the consequences. Although more research needs to be conducted to translate the present results to the field, one could think of several possible intervention strategies that could be derived from this perspective and that might differ in their adequateness depending on the specific circumstances. Strategies aimed at targets could be training to increase individual's social competences or teaching them self-organization and memory techniques. Strategies for sources could aim at sensitizing individuals to the negative consequences of ostracism to increase their empathy and tolerance for their fellow group member's weaknesses (Rudert \& Greifeneder, 2019). As for the situational circumstances, groups might be even less tolerant and inclusive when acting under limited time, resources and strong competition (Robinson et al., 2013). Thus, lowering competition and internal as well as external pressure on groups might be another way to foster intragroup inclusiveness.

\section{Conclusions}


In six studies, across four different paradigms, with participants from different cultural backgrounds, and with one nation-wide representative sample, we investigated the link between personality dispositions and ostracism. We find that especially low agreeableness and low conscientiousness were reliably associated with a higher risk of becoming a target of ostracism. The presented findings enhance our understanding why and under which conditions ostracism occurs in the first place, which is important to know when considering ways in which it may be prevented. 


\section{Footnotes}

${ }^{1}$ We analyzed the data with five single tests to allow for comparisons with the control group, which was the same for all personality dimensions. When excluding the control group, and analyzing the data with a $5 \times 2$ ANOVA (personality dimension x positivity-negativity), we find a significant interaction effect in both studies $F(4,637)=12.33, p<.001, \eta 2=.07$ and $F(4,728)$ $=22.36, p<.001, \eta 2=.11$, showing that the effect of positivity-negativity significantly differs across personality dimensions.

${ }^{2}$ Due to a programming error in the randomization, the manipulation check data could not be matched with the different group members. However, the data showed a significant main effect of the condition, $F(2,347)=66.88, p<.001, \eta^{2}=.28$, such that averaging together all three persons, mean conscientiousness was highest in the high conscientiousness condition and lowest in the low conscientiousness condition, all $p<.001$. When examining the data by condition, the pattern was in line with our assumptions (i.e., in the low conscientiousness condition, the group member that was rated lowest was rated substantially lower than the other two persons; and vice versa in the high conscientiousness condition). The programming error was corrected in Study 3b, and the Study $3 \mathrm{~b}$ manipulation proved fully successful. Given the almost identical set-up, we thus have reason to trust the manipulation in Study 3a as well. 


\section{References}

Ashton, M. C., Lee, K., \& Paunonen, S. V. (2002). What is the central feature of extraversion? Social attention versus reward sensitivity. Journal of Personality and Social Psychology, 83(1), 245 - 252. doi:10.1037/0022-3514.83.1.245

Barrick, M. R., \& Mount, M. K. (1991). The Big Five personality dimensions and job performance: A meta-analysis. Personnel Psychology, 44(1), 1-26. doi:10.1111/j.17446570.1991.tb00688.x

Berry, C. M., Ones, D. S., \& Sackett, P. R. (2007). Interpersonal deviance, organizational deviance, and their common correlates: A review and meta-analysis. Journal of Applied Psychology, 92(2), 410 - 424. doi:10.1037/0021-9010.92.2.410

Brähler, E., Mühlan, H., Albani, C., \& Schmidt, S. (2007). Teststatistische Prüfung und Normierung der deutschen Versionen des EUROHIS-QOL Lebensqualität-Index und des WHO-5 Wohlbefindens-Index. Diagnostica, 53(2), 83-96. doi:10.1026/00121924.53 .2 .83

Cain, S. (2013). Quiet: The power of introverts in a world that cant stop talking. London: Penguin Books.

Costa, P. T., \& McCrae, R. R. (1992). Four ways five factors are basic. Personality and Individual Differences, 13(6), 653-665. doi:10.1016/0191-8869(92)90236-I

Coyne, S. M., Nelson, D. A., Robinson, S. L., \& Gundersen, N. C. (2011). Is viewing ostracism on television distressing? The Journal of Social Psychology, 151(3), 213-217. doi:10.1080/00224540903365570 
Ditrich, L., \& Sassenberg, K. (2016). It's either you or me! Impact of deviations on social exclusion and leaving. Group Processes \& Intergroup Relations, 19(5), 630-652. doi:10.1177/1368430216638533

Downey, G., Freitas, A. L., Michaelis, B., \& Khouri, H. (1998). The self-fulfilling prophecy in close relationships: Rejection sensitivity and rejection by romantic partners. Journal of Personality and Social Psychology, 75(2), 545-560. doi:10.1037/0022-3514.75.2.545

Downey, G., Mougios, V., Ayduk, O., London, B. E., \& Shoda, Y. (2004). Rejection sensitivity and the defensive motivational system: Insights from the startle response to rejection cues. Psychological Science, 15(10), 668-673. doi:10.1111/j.0956-7976.2004.00738.x

Ferris, D. L., Brown, D. J., Berry, J. W., \& Lian, H. (2008). The development and validation of the Workplace Ostracism Scale. Journal of Applied Psychology, 93(6), 1348-1366. doi:10.1037/a0012743

Fiske, S. T. (1980). Attention and weight in person perception: The impact of negative and extreme behavior. Journal of Personality and Social Psychology, 38(6), 889-906. doi:10.1037/0022-3514.38.6.889

Fiske, S. T., Cuddy, A. J., \& Glick, P. (2007). Universal dimensions of social cognition: Warmth and competence. Trends in Cognitive Sciences, 11(2), 77-83. doi:10.1016/j.tics.2006.11.005

Fleeson, W. (2001). Toward a structure-and process-integrated view of personality: Traits as density distributions of states. Journal of Personality and Social Psychology, 80(6), 1011 -1027. doi:10.1037/0022-3514.80.6.1011 
Funk, F., Walker, M., \& Todorov, A. (2017). Modelling perceptions of criminality and remorse from faces using a data-driven computational approach. Cognition and Emotion, 31(7), 1431-1443. doi:10.1080/02699931.2016.1227305

Gelfand, M. J., Raver, J. L., Nishii, L., Leslie, L. M., Lun, J., Lim, B. C., . . Yamaguchi, S. (2011). Differences between tight and loose cultures: A 33-nation study. Science, 332(6033), 1100-1104. doi:10.1126/science.1197754

Gerlitz, J.-Y., \& Schupp, J. (2005). Zur Erhebung der Big-Five-basierten Persönlichkeitsmerkmale im SOEP. DIW Research Notes, 4.

Giner-Sorolla, R. (2018). Powering our interaction. Retrieved from https://approachingblog.wordpress.com/2018/01/24/powering-your-interaction-2/

Glaesmer, H., Grande, G., Braehler, E., \& Roth, M. (2011). The German version of the satisfaction with life scale (SWLS). European Journal of Psychological Assessment, 27(2), 127-132. doi:10.1027/1015-5759/a000058

Goebel, J., Grabka, M., Liebig, S., Kroh, M., Richter, D., Schröder, C., \& Schupp, J. (2018). The German Socio-Economic Panel (SOEP). Jahrbücher für Nationalökonomie und Statistik, 239(2), 345 - 360. doi:10.1515/jbnst-2018-0022

Graziano, W. G., \& Eisenberg, N. (1997). Agreeableness: A dimension of personality. In R. Hogan, J. Johnson, \& S. Briggs (Eds.), Handbook of Personality Psychology (pp. 795824). Cambridge, MA: Academic Press.

Graziano, W. G., Habashi, M. M., Sheese, B. E., \& Tobin, R. M. (2007). Agreeableness, empathy, and helping: A person $\times$ situation perspective. Journal of Personality and Social Psychology, 93(4), 583-599. doi:0.1037/0022-3514.93.4.583 
Güroğlu, B., Will, G.-J., \& Klapwijk, E. T. (2013). Some bullies are more equal than others: Peer relationships modulate altruistic punishment of bullies after observing ostracism. International Journal of Developmental Science, 7(1), 13-23. doi:10.3233/DEV-1312117

Hales, A. H., Kassner, M. P., Williams, K. D., \& Graziano, W. G. (2016). Disagreeableness as a cause and consequence of ostracism. Personality and Social Psychology Bulletin, 42(6), 782-797. doi:10.1177/0146167216643933

Hales, A. H., Wesselmann, E. D., \& Williams, K. D. (2016). Prayer, self-affirmation, and distraction improve recovery from short-term ostracism. Journal of Experimental Social Psychology, 64, 8-20. doi:http://dx.doi.org/10.1016/j.jesp.2016.01.002

Hawkley, L. C., Duvoisin, R., Ackva, J., Murdoch, J. C., \& Luhmann, M. (2015). Loneliness in older adults in the USA and Germany: Measurement invariance and validation. Retrieved from http://www.norc.org/PDFs/Working\%20Paper\%20Series/WP-2015004.pdf

Hayes, A. F. (2013). Introduction to mediation, moderation and conditional process analysis. New York, NY: Guilford.

Hofstede, G. (1980). Culture's consequences. Beverly Hills: Sage Publications.

Hurtz, G. M., \& Donovan, J. J. (2000). Personality and job performance: The Big Five revisited. Journal of Applied Psychology, 85(6), 869-875. doi:10.1037/0021-9010.85.6.869

Jensen-Campbell, L. A., Adams, R., Perry, D. G., Workman, K. A., Furdella, J. Q., \& Egan, S. K. (2002). Agreeableness, extraversion, and peer relations in early adolescence: Winning friends and deflecting aggression. Journal of Research in Personality, 36(3), 224-251. doi:10.1006/jrpe.2002.2348 
Kagel, J., \& McGee, P. (2014). Personality and cooperation in finitely repeated prisoner's dilemma games. Economics Letters, 124(2), 274-277. doi:10.1016/j.econlet.2014.05.034

Keller, M. D., Reutner, L., Greifeneder, R., \& Walker, M. (2019). Faces evoking emotions stereotypically triggered by Groups: Developing an advanced Reverse Correlation Technique. Manuscript under review.

Kerr, N. L., \& Levine, J. M. (2008). The detection of social exclusion: Evolution and beyond. Group Dynamics: Theory, Research, and Practice, 12(1), 39-52. doi:10.1037/10892699.12.1.39

Kervyn, N., Yzerbyt, V. Y., Demoulin, S., \& Judd, C. M. (2008). Competence and warmth in context: The compensatory nature of stereotypic views of national groups. European Journal of Social Psychology, 38(7), 1175-1183. doi:10.1002/ejsp.526

Koch, A., Imhoff, R., Dotsch, R., Unkelbach, C., \& Alves, H. (2016). The ABC of stereotypes about groups: Agency/socioeconomic success, conservative-progressive beliefs, and communion. Journal of Personality and Social Psychology, 110(5), 675-709. doi: $10.1037 /$ pspa0000046

Kurzban, R., \& Leary, M. R. (2001). Evolutionary origins of stigmatization: The functions of social exclusion. Psychological Bulletin, 127(2), 187-208. doi:10.1037/00332909.127.2.187

Lang, F. R., Weiss, D., Gerstorf, D., \& Wagner, G. G. (2013). Forecasting life satisfaction across adulthood: Benefits of seeing a dark future? Psychology and Aging, 28(1), 249-261. doi:10.1037/a0030797 
Legate, N., DeHaan, C. R., Weinstein, N., \& Ryan, R. M. (2013). Hurting you hurts me too: The psychological costs of complying with ostracism. Psychological Science, 24(4), 583-588. doi:10.1177/0956797612457951

Leising, D., Erbs, J., \& Fritz, U. (2010). The letter of recommendation effect in informant ratings of personality. Journal of Personality and Social Psychology, 98(4), 668-682. doi:10.1037/a0018771

Lewicki, P. (1983). Self-image bias in person perception. Journal of Personality and Social Psychology, 45(2), 384 - 393. doi:45(2), 384-393.

$10.1037 / 0022-3514.45 .2 .384$

Lindström, B., \& Tobler, P. N. (2018). Incidental ostracism emerges from simple learning mechanisms. Nature Human Behaviour, 405-414. doi:10.1038/s41562-018-0355-y

Luhmann, M., \& Hawkley, L. C. (2016). Age differences in loneliness from late adolescence to oldest old age. Developmental Psychology, 52(6), 943. doi:10.1037/dev0000117

Maner, J. K., \& Mead, N. L. (2010). The essential tension between leadership and power: When leaders sacrifice group goals for the sake of self-interest. Journal of Personality and Social Psychology, 99(3), 482-497. doi:10.1037/a0018559

Mangini, M. C., \& Biederman, I. (2004). Making the ineffable explicit: Estimating the information employed for face classifications. Cognitive Science, 28(2), 209-226. doi: $10.1207 / \mathrm{s} 15516709 \operatorname{cog} 2802 \_4$

Markus, H. R., \& Kitayama, S. (1991). Culture and the self: Implications for cognition, emotion, and motivation. Psychological Review, 98(2), 224 - 253. doi:10.1037//0033$295 \times .98 .2 .224$ 
Masten, C. L., Eisenberger, N. I., Pfeifer, J. H., \& Dapretto, M. (2010). Witnessing peer rejection during early adolescence: Neural correlates of empathy for experiences of social exclusion. Social neuroscience, 5(5-6), 496-507. doi:10.1080/17470919.2010.490673

McCrae, R. R., \& John, O. P. (1992). An introduction to the five-factor model and its applications. Journal of Personality, 60(2), 175-215. doi:10.1111/j.14676494.1992.tb00970.x

McCrae, R. R., \& Sutin, A. R. (2009). Openness to experience. In M. R. Leary \& R. H. Hoyle (Eds.), Handbook of Individual Differences in Social Behavior (Vol. 15, pp. 257-273). New York, NY: Guilford Publications.

McGrath, J. E. (1984). Groups: Interaction and performance (Vol. 14). Englewood Cliffs, NJ: Prentice-Hall

Milam, A. C., Spitzmueller, C., \& Penney, L. M. (2009). Investigating individual differences among targets of workplace incivility. Journal of Occupational Health Psychology, 14(1), 58-69. doi:10.1037/a0012683

Nezlek, J. B., Wesselmann, E. D., Wheeler, L., \& Williams, K. D. (2012). Ostracism in everyday life. Group Dynamics: Theory, Research, and Practice, 16(2), 91-104. doi:10.1037/a0028029

Nezlek, J. B., Wesselmann, E. D., Wheeler, L., \& Williams, K. D. (2015). Ostracism in everyday life: The effects of ostracism on those who ostracize. The Journal of Social Psychology, 155(5), 432-451. doi:10.1080/00224545.2015.1062351

Nielsen, M. B., Glasø, L., \& Einarsen, S. (2017). Exposure to workplace harassment and the Five Factor Model of personality: A meta-analysis. Personality and Individual Differences, 104, 195-206. doi:10.1016/j.paid.2016.08.015 
Nielsen, M. B., \& Knardahl, S. (2015). Is workplace bullying related to the personality traits of victims? A two year prospective study. Work \& Stress, 29(2), 128-149. doi:10.1080/02678373.2015.1032383

Nisbett, R. E., \& Wilson, T. D. (1977). The halo effect: Evidence for unconscious alteration of judgments. Journal of Personality and Social Psychology, 35(4), 250-256. doi:10.1037/0022-3514.35.4.250

O’Connor, M. C., \& Paunonen, S. V. (2007). Big Five personality predictors of post-secondary academic performance. Personality and Individual Differences, 43(5), 971-990. doi:10.1016/j.paid.2007.03.017

Olivola, C. Y., \& Todorov, A. (2010). Fooled by first impressions? Reexamining the diagnostic value of appearance-based inferences. Journal of Experimental Social Psychology, 46(2), 315-324. doi:10.1016/j.jesp.2009.12.002

Over, H., \& Uskul, A. K. (2016). Culture moderates children's responses to ostracism situations. Journal of Personality and Social Psychology, 110(5), 710 - 724. doi:10.1037/pspi0000050

Pallesen, S., Nielsen, M. B., Mageroy, N., Andreassen, C. S., \& Einarsen, S. (2017). An experimental study on the attribution of personality traits to bullies and targets in a workplace setting. Frontiers in Psychology, 8. doi:10.3389/fpsyg.2017.01045

Paulhus, D. L. (1998). Interpersonal and intrapsychic adaptiveness of trait self-enhancement: A mixed blessing? Journal of Personality and Social Psychology, 74(5), 1197 - 1208. doi:10.1037/0022-3514.74.5.1197 
Paulhus, D. L., \& Vazire, S. (2007). The self-report method. In R. W. Robins, R. C. Fraley, \& R. F. Krueger (Eds.), Handbook of research methods in personality psychology (pp. 225239). New York, NY: Guilford Press.

Paysan, P., Knothe, R., Amberg, B., Romdhani, S., \& Vetter, T. (2009). A 3D face model for pose and illumination invariant face recognition. 6th IEEE International Conference on Advanced Video and Signal based Surveillance for Security, Safety and Monitoring in Smart Environments, Italy, 296 - 301. Retrieved from http://gravis.cs.unibas.ch/publications/2009/BFModel09.pdf

Podsiadly, A., \& Gamian-Wilk, M. (2017). Personality traits as predictors or outcomes of being exposed to bullying in the workplace. Personality and Individual Differences, 115, 43-49. doi:10.1016/j.paid.2016.08.001

Poropat, A. E. (2009). A meta-analysis of the five-factor model of personality and academic performance. Psychological Bulletin, 135(2), 322-338. doi:10.1037/a0014996

Rammstedt, B., \& John, O. P. (2005). Kurzversion des Big Five Inventory (BFI-K). Diagnostica, 51(4), 195-206. doi:10.1026/0012-1924.51.4.195

Ren, D., Wesselmann, E., \& Williams, K. D. (2016). Evidence for another response to ostracism: Solitude seeking. Social Psychological and Personality Science, 7(3), 204-212. doi:10.1177/1948550615616169

Robinson, S. L., O’Reilly, J., \& Wang, W. (2013). Invisible at work: An integrated model of workplace ostracism. Journal of Management, 39(1), 203-231.

doi:10.1177/0149206312466141 
Rothmann, S., \& Coetzer, E. P. (2003). The big five personality dimensions and job performance. SA Journal of Industrial Psychology, 29(1), 68-74. doi:10.4102/sajip.v29i1.88

Rudert, S. C., \& Greifeneder, R. (2016). When it's okay that I don't play: Social norms and the situated construal of social exclusion. Personality and Social Psychology Bulletin, 42(7), 955-969. doi:10.1177/0146167216649606

Rudert, S. C., \& Greifeneder, R. (2019). Observing Ostracism: How observers interpret and respond to ostracism situations In S. C. Rudert, R. Greifeneder, \& K. D. Williams (Eds.), Current Directions in Ostracism, Social Exclusion and Rejection (pp. 136-154). Oxford, UK: Routledge.

Rudert, S. C., Hales, A. H., Greifeneder, R., \& Williams, K. D. (2017). When silence is not golden: Why acknowledgement matters even when being excluded. Personality and Social Psychology Bulletin, 43(5), 678 - 692. doi:10.1177/0146167217695554

Rudert, S. C., Reutner, L., Greifeneder, R., \& Walker, M. (2017). Faced with exclusion: Perceived facial warmth and competence influence moral judgments of social exclusion. Journal of Experimental Social Psychology, 68, 101-112. doi:10.1016/j.jesp.2016.06.005

Rudert, S. C., Ruf, S., \& Greifeneder, R. (in press). Who to punish? How observers sanction norm-violating behavior in ostracism situations. European Journal of Social Psychology.

Rudert, S. C., Sutter, D., Corrodi, C., \& Greifeneder, R. (2018). Who's to blame? Dissimilarity as a cue in moral judgments of observed ostracism episodes. Journal of Personality and Social Psychology, 115, 31-53. doi:10.1037/pspa0000122

Runge, T. E., Frey, D., Gollwitzer, P. M., Helmreich, R. L., \& Spence, J. T. (1981). Masculine (instrumental) and feminine (expressive) traits: A comparison between students in the 
United States and West Germany. Journal of Cross-Cultural Psychology, 12(2), 142-162. doi:10.1177/0022022181122002

Samuel, D. B., \& Widiger, T. A. (2011). Conscientiousness and obsessive-compulsive personality disorder. Personality Disorders: Theory, Research, Treatment, 2(3), 161-174. doi:10.1037/a0021216

Scheepers, D., Branscombe, N. R., Spears, R., \& Doosje, B. (2002). The emergence and effects of deviants in low and high status groups. Journal of Experimental Social Psychology, 38(6), 611-617. doi:10.1016/S0022-1031(02)00506-1

Schimmack, U., Krause, P., Wagner, G. G., \& Schupp, J. (2010). Stability and change of well being: An experimentally enhanced latent state-trait-error analysis. Social Indicators Research, 95(1), 19-31. doi:10.1007/s11205-009-9443-8

Schmitt, M., Baumert, A., Gollwitzer, M., \& Maes, J. (2010). The Justice Sensitivity Inventory: Factorial validity, location in the personality facet space, demographic pattern, and normative data. Social Justice Research, 23(2-3), 211-238. doi:10.1007/s11211-0100115-2

Sheldon, K. M., \& Hilpert, J. C. (2012). The balanced measure of psychological needs (BMPN) scale: An alternative domain general measure of need satisfaction. Motivation and Emotion, 36(4), 439-451. doi:10.1007/s11031-012-9279-4

Simonsohn, U. (Producer). (2014). No-way interactions. Retrieved from http://datacolada.org/17 Skowronski, J. J., \& Carlston, D. E. (1989). Negativity and extremity biases in impression formation: A review of explanations. Psychological Bulletin, 105(1), 131-142. doi:10.1037/0033-2909.105.1.131 
Snyder, M., \& Gangestad, S. (1982). Choosing social situations: Two investigations of selfmonitoring processes. Journal of Personality and Social Psychology, 43(1), 123-135. doi:10.1037/0022-3514.43.1.123

Soto, C. J., \& John, O. P. (2017). The next Big Five Inventory (BFI-2): Developing and assessing a hierarchical model with 15 facets to enhance bandwidth, fidelity, and predictive power. Journal of Personality and Social Psychology, 113(1), 117-143. doi:10.1037/pspp0000096

Stolier, R. M., Hehman, E., Keller, M. D., Walker, M., \& Freeman, J. B. (2018). The conceptual structure of face impressions. Proceedings of the National Academy of Sciences, 115(37), 9210 - 9215. doi:10.1073/pnas.1807222115

Timeo, S., Riva, P., \& Paladino, M. P. (2019). Dealing with social exclusion: An analysis of psychological strategies to deal with social exclusion. In S. C. Rudert, R. Greifeneder, \& K. D. Williams (Eds.), Current Directions in Ostracism, Social Exclusion and Rejection Research. London, UK: Routledge.

Twenge, J. M., Baumeister, R. F., DeWall, C. N., Ciarocco, N. J., \& Bartels, J. M. (2007). Social exclusion decreases prosocial behavior. Journal of Personality and Social Psychology, 92(1), 56-66. doi:10.1037/0022-3514.92.1.56

van Geel, M., Goemans, A., Toprak, F., \& Vedder, P. (2017). Which personality traits are related to traditional bullying and cyberbullying? A study with the Big Five, Dark Triad and sadism. Personality and Individual Differences, 106, 231-235.

Vazire, S. (2010). Who knows what about a person? The self-other knowledge asymmetry (SOKA) model. Journal of Personality and Social Psychology, 98(2), 281-300. doi:10.1037/a0017908 
Vetter, T., \& Walker, M. (2011). Computer-generated images in face perception. In A. Calder, J. V. Haxby, M. Johnson, \& G. Rhodes (Eds.), The Oxford Handbook of Face Perception (pp. 388-399). Oxford, UK: Oxford University Press.

Walker, M., Jiang, F., Vetter, T., \& Sczesny, S. (2011). Universals and cultural differences in forming personality trait judgments from faces. Social Psychological and Personality Science, 2(6), 609-617. doi:10.1177/1948550611402519

Walker, M., \& Keller, M. D. (in press). Beyond attractiveness: A multi-method approach to study enhancement in self-recognition on the Big Two personality dimensions. Journal of Personality and Social Psychology. doi:10.1037/pspa0000157

Walker, M., Schönborn, S., Greifeneder, R., \& Vetter, T. (2018). The Basel Face Database: A validated set of photographs reflecting systematic differences in Big Two and Big Five personality dimensions. PloS one, 13(3), e0193190. doi:10.1371/journal.pone.0193190

Walker, M., \& Vetter, T. (2016). Changing the personality of a face: Perceived Big Two and Big Five personality factors modeled in real photographs. Journal of Personality and Social Psychology, 110(4), 609-624. doi:10.1037/pspp0000064

Wesselmann, E. D., Bagg, D., \& Williams, K. D. (2009). "I feel your pain": The effects of observing ostracism on the ostracism detection system. Journal of Experimental Social Psychology, 45(6), 1308-1311. doi:10.1016/j.jesp.2009.08.003

Wesselmann, E. D., Williams, K. D., \& Wirth, J. H. (2014). Ostracizing group members who can (or cannot) control being burdensome. Human Ethology Bulletin, 29(2), 82-103.

Wesselmann, E. D., Wirth, J. H., Pryor, J. B., Reeder, G. D., \& Williams, K. D. (2013). When do we ostracize? Social Psychological and Personality Science, 4(1), 108-115. doi:10.1177/1948550612443386 
Wesselmann, E. D., Wirth, J. H., Pryor, J. B., Reeder, G. D., \& Williams, K. D. (2015). The role of burden and deviation in ostracizing others. The Journal of Social Psychology, 155(5), 483-496. doi:10.1080/00224545.2015.1060935

Will, G.-J., Crone, E. A., van den Bos, W., \& Güroğlu, B. (2013). Acting on observed social exclusion: Developmental perspectives on punishment of excluders and compensation of victims. Developmental Psychology, 49(12), 2236-2244. doi:10.1037/a0032299

Williams, K. D. (1997). Social ostracism. In R. M. Kowalski (Ed.), Aversive Interpersonal Behaviors. The Social/Clinical Psychology (pp. 133-170). Boston, MA: Springer.

Williams, K. D. (2009). Ostracism: A temporal need-threat model. In P. Z. Mark (Ed.), Advances in experimental social psychology (Vol. 41, pp. 275-314). San Diego, CA: Elsevier Academic Press.

Wirth, J. H., Lynam, D. R., \& Williams, K. D. (2010). When social pain is not automatic: Personality disorder traits buffer ostracism's immediate negative impact. Journal of Research in Personality, 44(3), 397-401. doi:10.1016/j.jrp.2010.03.001

Wu, L., Wei, L., \& Hui, C. (2011). Dispositional antecedents and consequences of workplace ostracism: An empirical examination. Frontiers of Business Research in China, 5(1), 2344. doi:10.1007/s11782-011-0119-2

Zadro, L., Boland, C., \& Richardson, R. (2006). How long does it last? The persistence of the effects of ostracism in the socially anxious. Journal of Experimental Social Psychology, 42(5), 692-697. doi:10.1016/j.jesp.2005.10.007 
Table 1

Manipulation of the Personality Dimensions in Studies 1 and 2

\begin{tabular}{lll}
\hline Personality Dimension & \multicolumn{1}{c}{ Low } & High \\
Conscientiousness & $\begin{array}{l}\text { Mason tends to be a lazy, chaotic person } \\
\text { and an unreliable and careless worker. }\end{array}$ & $\begin{array}{l}\text { Mason tends to be a diligent, well- } \\
\text { organized person and a reliable and } \\
\text { precise worker. }\end{array}$ \\
\hline Agreeableness & $\begin{array}{l}\text { Mason tends to be a cold, untrusting, and } \\
\text { uncaring person. }\end{array}$ & $\begin{array}{l}\text { Mason tends to be a warm, trusting, and } \\
\text { caring person. }\end{array}$ \\
\hline Neuroticism & $\begin{array}{l}\text { Mason tends to be a relaxed, confident, } \\
\text { and cheerful person. }\end{array}$ & $\begin{array}{l}\text { Mason tends to be an anxious, insecure, } \\
\text { and moody person. }\end{array}$ \\
\hline Openness & $\begin{array}{l}\text { Mason tends to be an unimaginative } \\
\text { person who likes to think in familiar } \\
\text { patterns and prefers to do things in a } \\
\text { routine way. }\end{array}$ & $\begin{array}{l}\text { Mason tends to be an ingenious person } \\
\text { who likes to come up with new ideas and } \\
\text { prefers to do things in an inventive way. }\end{array}$ \\
\hline $\begin{array}{l}\text { Mason tends to be a quiet, calm, and } \\
\text { reserved person. }\end{array}$ & $\begin{array}{l}\text { Mason tends to be a talkative, energetic, } \\
\text { and outgoing person. }\end{array}$ \\
\hline
\end{tabular}


Table 2

Means (and standard deviations) of the manipulation checks as a function of the manipulated personality dimension in Study 1.

\begin{tabular}{lccc}
\hline Personality Dimension & Low & Control & High \\
\hline Conscientiousness & $1.65(1.36)^{\mathrm{a}}$ & $5.29(1.34)^{\mathrm{b}}$ & $6.50(.86)^{\mathrm{c}}$ \\
\hline Agreeableness & $2.85(1.92)^{\mathrm{a}}$ & $5.62(1.17)^{\mathrm{b}}$ & $6.58(.70)^{\mathrm{c}}$ \\
\hline Neuroticism & $1.69(.98)^{\mathrm{a}}$ & $2.49(1.24)^{\mathrm{b}}$ & $5.22(1.47)^{\mathrm{c}}$ \\
\hline Openness & $3.42(1.76)^{\mathrm{a}}$ & $5.13(1.39)^{\mathrm{b}}$ & $5.96(1.18)^{\mathrm{c}}$ \\
\hline Extraversion & $2.28(1.45)^{\mathrm{a}}$ & $3.63(1.66)^{\mathrm{b}}$ & $5.57(1.82)^{\mathrm{c}}$ \\
\hline
\end{tabular}

Note. The letters a - b represent significant differences between groups; all values in the same row that share the same letter do not differ significantly from each other, values with different letters do. 


\section{Table 3}

Means (and standard deviations) of participants' intention to ostracize Mason as a function of the manipulated personality dimension in Study 1

\begin{tabular}{lccc}
\hline Personality Dimension & Low & Control & High \\
\hline Conscientiousness & $2.58(.87)^{\mathrm{a}}$ & $1.67(.73)^{\mathrm{b}}$ & $1.54(.60)^{\mathrm{b}}$ \\
\hline Agreeableness & $2.65(.92)^{\mathrm{a}}$ & $1.67(.73)^{\mathrm{b}}$ & $1.61(.68)^{\mathrm{b}}$ \\
\hline Neuroticism & $1.52(.64)^{\mathrm{a}}$ & $1.67(.73)^{\mathrm{a}}$ & $2.10(.78)^{\mathrm{b}}$ \\
\hline Openness & $2.03(.73)^{\mathrm{a}}$ & $1.67(.73)^{\mathrm{b}}$ & $1.68(.67)^{\mathrm{b}}$ \\
\hline Extraversion & $1.68(.60)^{\mathrm{a}}$ & $1.67(.73)^{\mathrm{a}}$ & $1.69(.75)^{\mathrm{a}}$ \\
\hline
\end{tabular}

Note. Note that the control group was the same for all personality dimensions (no information about personality provided). The letters $\mathrm{a}-\mathrm{b}$ represent significant differences between groups; all values in the same row that share the same letter do not differ significantly from each other, values with different letters do. 
Table 4

Means (and standard deviations) of the manipulation checks as a function of the manipulated personality dimension in Study 2.

\begin{tabular}{lccc}
\hline Personality Dimension & Low & Control & High \\
\hline Conscientiousness & $1.33(.55)^{\mathrm{a}}$ & $5.11(1.13)^{\mathrm{b}}$ & $6.50(.73)^{\mathrm{c}}$ \\
\hline Agreeableness & $2.88(1.68)^{\mathrm{a}}$ & $5.13(1.14)^{\mathrm{b}}$ & $6.42(.84)^{\mathrm{c}}$ \\
\hline Neuroticism & $1.96(1.14)^{\mathrm{a}}$ & $2.89(1.43)^{\mathrm{b}}$ & $5.08(1.73)^{\mathrm{c}}$ \\
\hline Openness & $3.19(1.57)^{\mathrm{a}}$ & $4.69(.93)^{\mathrm{b}}$ & $6.15(1.06)^{\mathrm{c}}$ \\
\hline Extraversion & $2.19(1.04)^{\mathrm{a}}$ & $3.33(1.35)^{\mathrm{b}}$ & $5.90(1.66)^{\mathrm{c}}$ \\
\hline
\end{tabular}

Note. The letters a - b represent significant differences between groups; all values in the same row that share the same letter do not differ significantly from each other, values with different letters do. 


\section{Table 5}

Means (and standard deviations) of participants' intention to ostracize Mason as a function of the manipulated personality dimension in Study 2

\begin{tabular}{lllll}
\hline Personality Dimension & Dependent Variable & Low & Control & High \\
\hline \multirow{2}{*}{ Conscientiousness } & Ostracism Intentions & $2.60(.83)^{\mathrm{a}}$ & $1.59(.65)^{\mathrm{b}}$ & $1.52(.69)^{\mathrm{b}}$ \\
& Liking & $2.77(.71)^{\mathrm{a}}$ & $3.62(.59)^{\mathrm{b}}$ & $3.88(.59)^{\mathrm{c}}$ \\
\hline \multirow{2}{*}{ Agreeableness } & Ostracism Intentions & $2.72(.94)^{\mathrm{a}}$ & $1.59(.65)^{\mathrm{b}}$ & $1.47(.44)^{\mathrm{b}}$ \\
& Liking & $2.67(.84)^{\mathrm{a}}$ & $3.62(.59)^{\mathrm{b}}$ & $3.87(.62)^{\mathrm{b}}$ \\
\hline \multirow{2}{*}{ Neuroticism } & Ostracism Intentions & $1.52(.63)^{\mathrm{a}}$ & $1.59(.65)^{\mathrm{a}}$ & $1.88(.75)^{\mathrm{b}}$ \\
& Liking & $3.81(.61)^{\mathrm{a}}$ & $3.62(.59)^{\mathrm{a}}$ & $3.27(.77)^{\mathrm{b}}$ \\
\hline \multirow{2}{*}{ Openness } & Ostracism Intentions & $1.87(.70)^{\mathrm{a}}$ & $1.59(.65)^{\mathrm{b}}$ & $1.57(.63)^{\mathrm{b}}$ \\
& Liking & $3.39(.68)^{\mathrm{a}}$ & $3.62(.59)^{\mathrm{a}}$ & $3.95(.57)^{\mathrm{b}}$ \\
\hline \multirow{2}{*}{ Extraversion } & Ostracism Intentions & $1.66(.61)^{\mathrm{a}}$ & $1.59(.65)^{\mathrm{a}}$ & $1.68(.64)^{\mathrm{a}}$ \\
& Liking & $3.79(.55)^{\mathrm{a}}$ & $3.62(.59)^{\mathrm{a}}$ & $3.75(.62)^{\mathrm{a}}$ \\
\hline
\end{tabular}

Note. Note that the control group was the same for all personality dimensions (no information about personality provided). The letters $\mathrm{a}-\mathrm{b}$ represent significant differences between groups; all values in the same row that share the same letter do not differ significantly from each other, values with different letters do. 
Table 6

Validity of the Ostracism Short Scale

$\begin{array}{lllllllll}(1) & (2) & (3) & (4) & (5) & (6) & (7) & (8) & \text { (9) }\end{array}$

(1) Ostracism Short Scale

(2) Life Satisfaction $\quad \mathbf{- . 5 0}^{\text {** }}$

(3) Life Satisfaction (SOEP) $\quad-. .49^{* *} \quad .78^{* *}$

(4) Life Satisfaction 1 y (SOEP) $\quad-.44^{* *} \quad .67^{* *} \quad .80^{* *}$

(5) Life Satisfaction 5 y (SOEP) $\quad-. .34^{* *} \quad .65^{* *} \quad .71^{* *} \quad .86^{* *}$

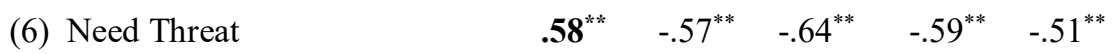

(7) Mood

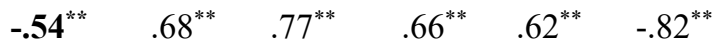

(8) Well-being

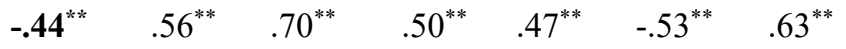

(9) Relatedness

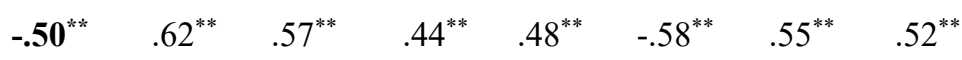

(10) Loneliness

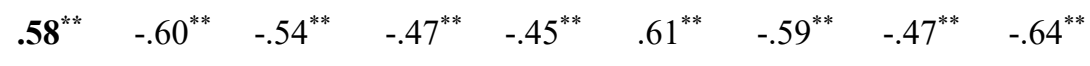

${ }^{* * *} \mathrm{p}<.001$ 


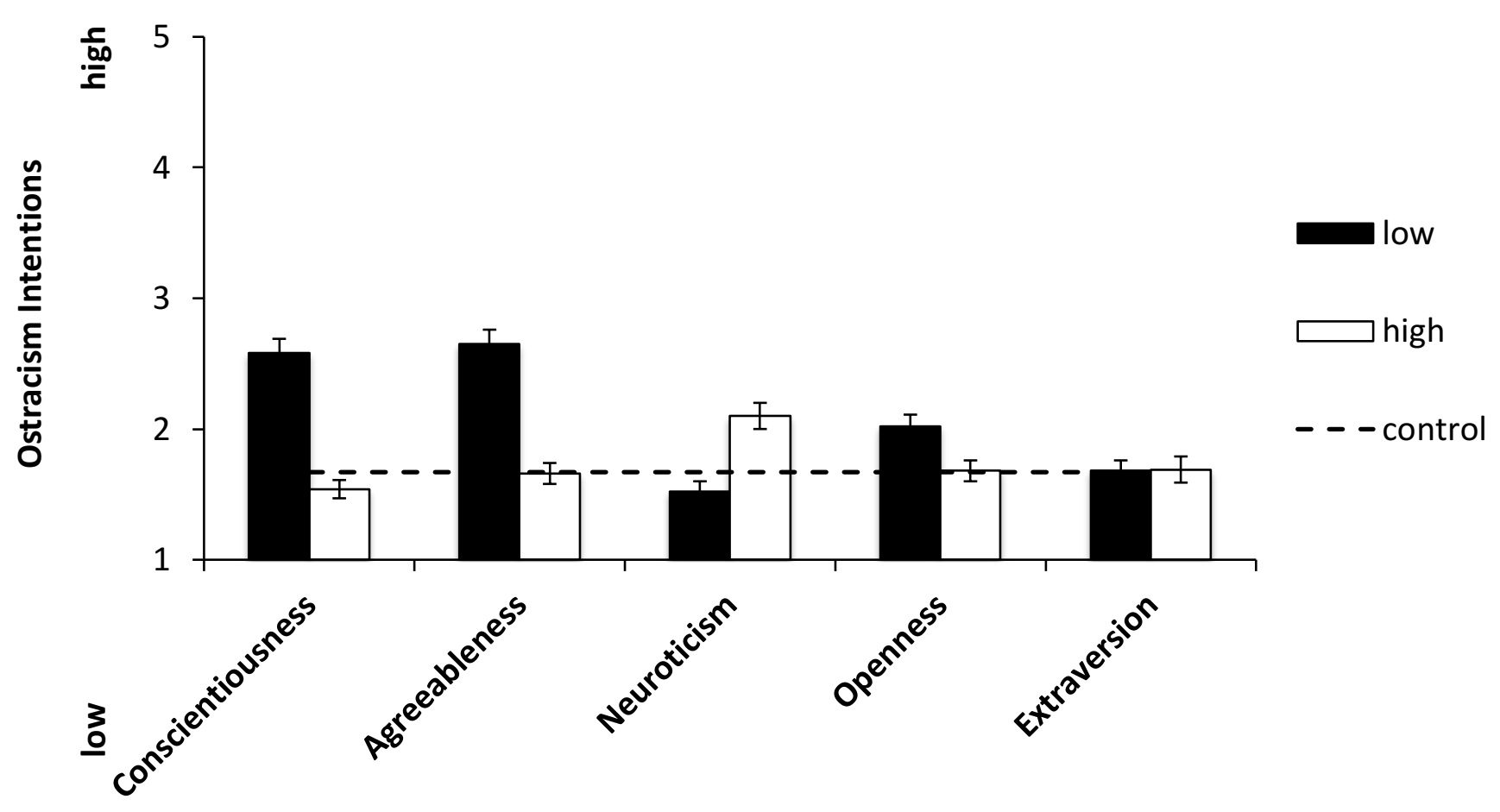

Figure 1. Mean ostracism intentions (with standard errors) as a function of the manipulated personality dimension in Study 2. The control condition mean is displayed as a dotted line. 
$\mathbf{a}$

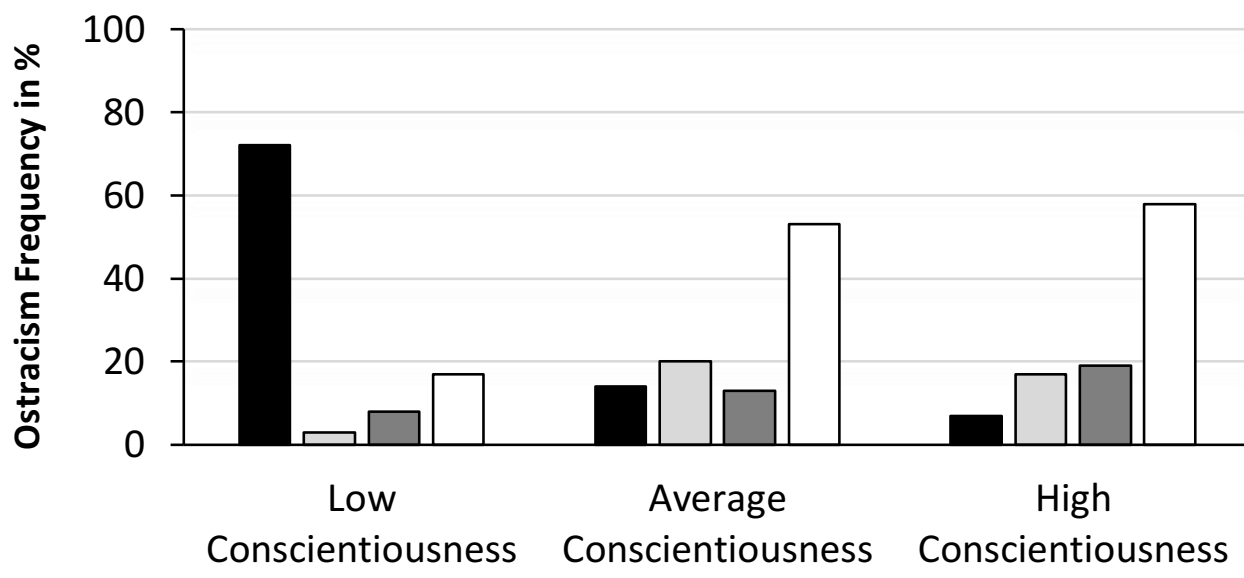

- Target

$\square$ Control 1

$\square$ Control 2

$\square$ No one

b

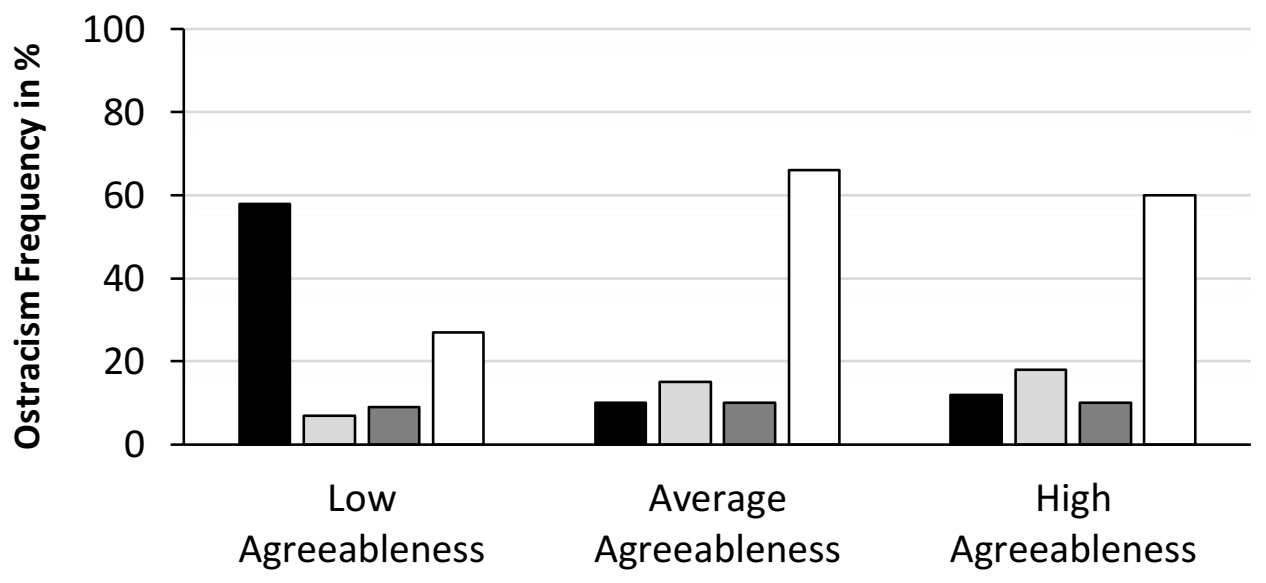

- Target

$\square$ Control 1

$\square$ Control 2

$\square$ No one

Figure 2. Ostracism frequency in percent as a function of the manipulated personality dimension (a: conscientiousness, b: agreeableness) in Studies $3 \mathrm{a}$ and $\mathrm{b}$. 


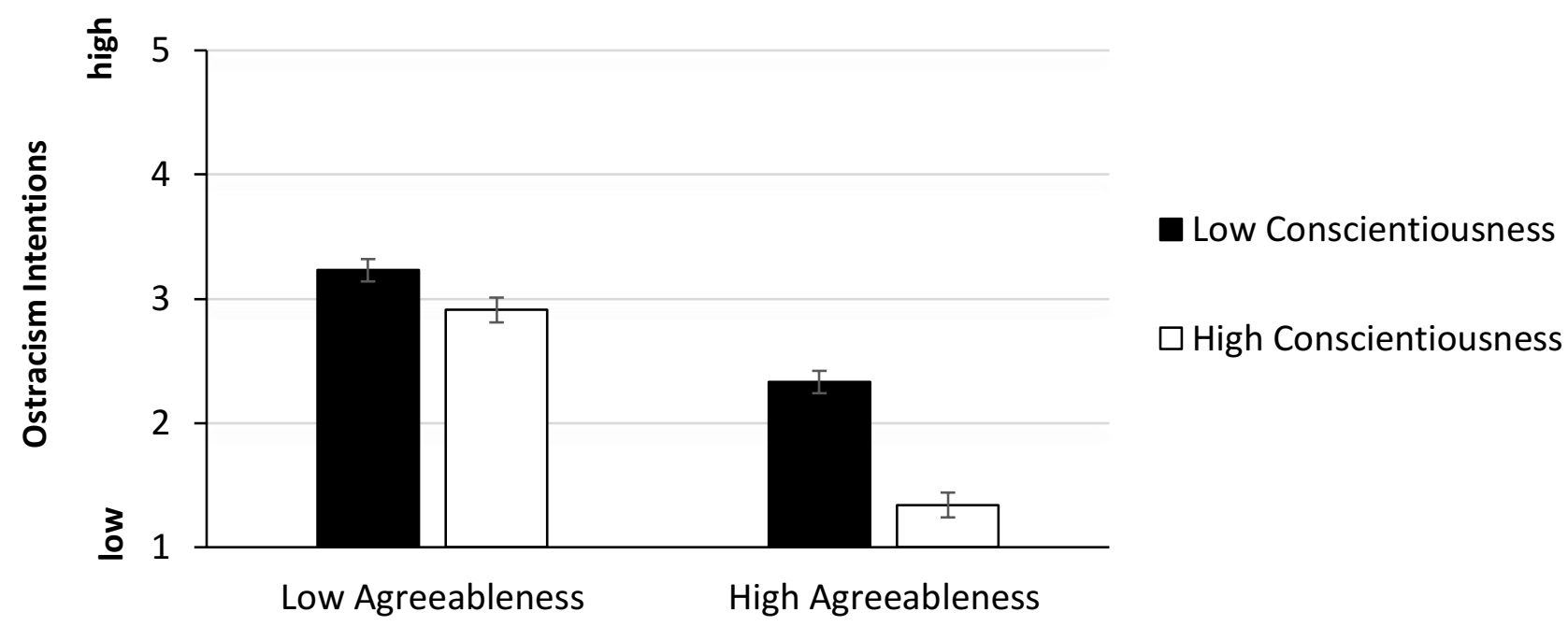

Figure 3. Mean ostracism intentions (with standard errors) as a function of manipulated agreeableness and conscientiousness in Study 4. 


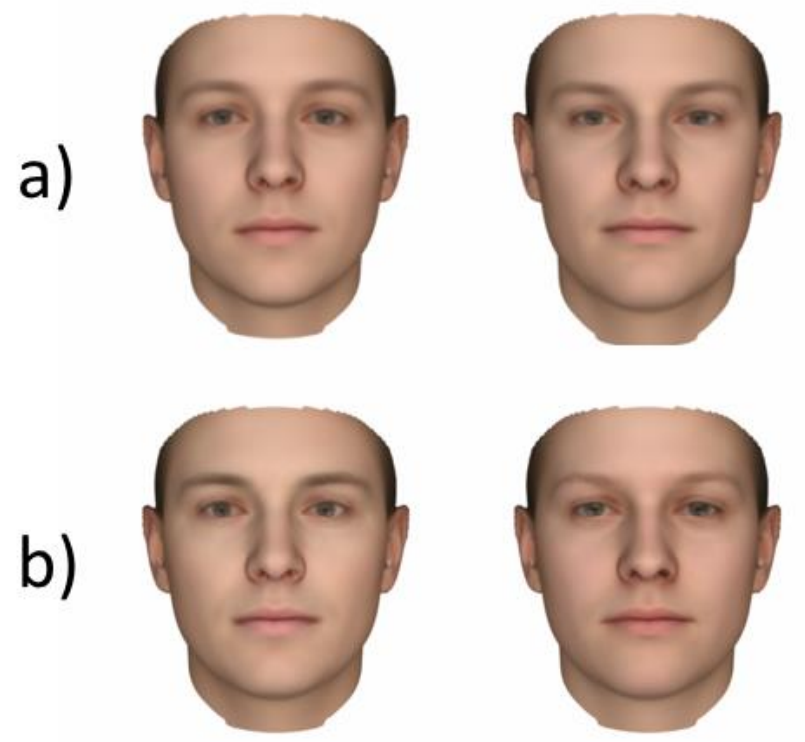

Figure 4. Two exemplar face pairs as presented in the image classification trials in Study 5a visualizing a) two faces only varying regarding shape information and b) two faces only varying regarding color information. 

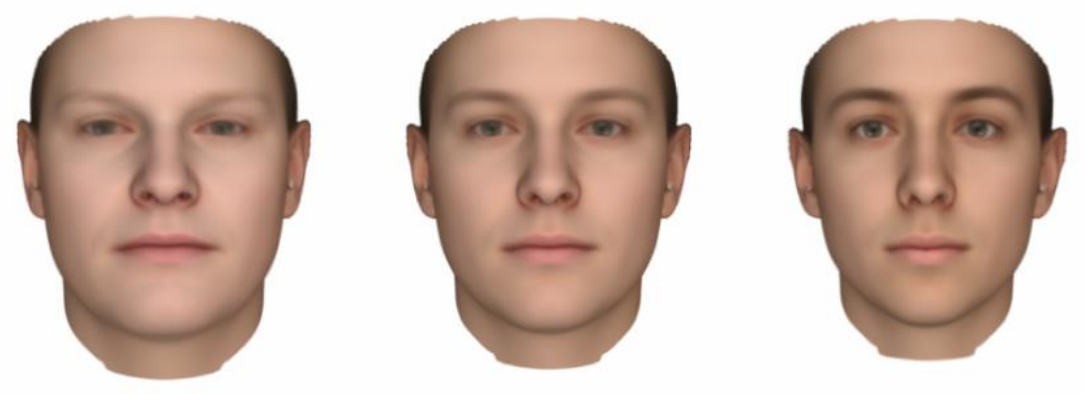

Figure 5. Visualization of the Ostracism facial stereotype (left), the average face from the Basel Face Model (middle), and the Anti-Ostracism facial stereotype (i.e., the face pointing in the opposite direction from the average face; right) as extracted in Study 5a. 